\title{
Detonator Cable Initiation System Safety Investigation: Consequences of Energizing the Detonator and Actuator Cables
}

\author{
J. Osher \\ H. Chau \\ W. Von Holle
}

March 1994 


\section{DISCLAIMER}

This document was prepared as an account of work sponsored by an agency of the United States Government. Nelther the United States Government nor the University of California nor any of their employees, makes any Farranty, express or implied, or assumes any legal liability or responsibility for the accuracy, completeness, or usefulness of any information, apparatus, product, or process disclosed, or represents that its use would not infringe privately owned rights. Reference herein to any specific commercial products, process, or service by trade name, trademark, manufacturer, or otherwise, does not necessarily constitute or imply its endorsement, recommendation, or favoring by the United States Government or the University of California. The views and opinions of authors expressed herein do not necessarily state or reflect those of the United States Government or the University of California, and shall not be used for advertising or product endorsement purposes.

This report has been reproduced directly from the best available copy.

A vailable to DOE and DOE contractors from the Omice of Scientific and Technical Informution

P.O. Box 62, Oak Ridge, TN 37831

Prices available from (615) 576-8401, FTS 626-8401

Available to the public from the National Technical Information Service

U.S. Department of Commerce 5285 Port Royal Rd. Springrield, VA 22161 
UCRL-LR-115294

Distribution Category UC-742

\title{
Detonator Cable Initiation System Safety Investigation: Consequences of Energizing the Detonator and Actuator Cables
}

\author{
J. Osher \\ H. Chau \\ W. Von Holle
}

Manuscript date: March 1994

LAWRENCE LIVERMORE NATIONAL LABORATORY University of California - Livermore, California • 94551 


\section{CONTENTS}

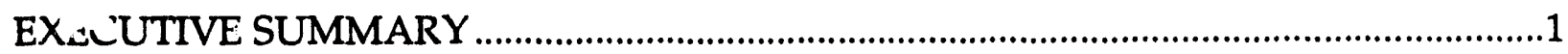

INTRODUCTION

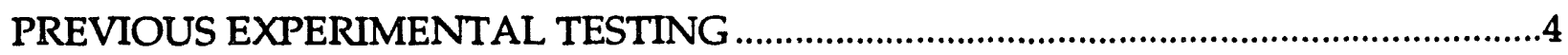

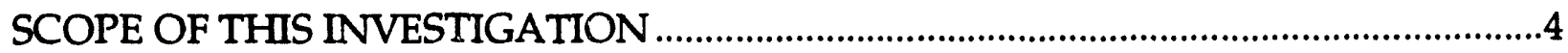

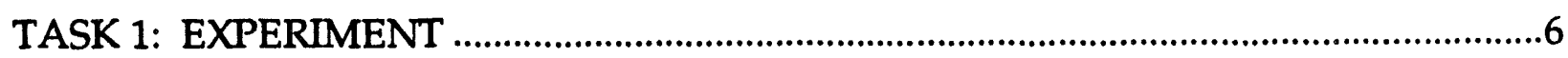

Detonator-Cable Explosion Launch of "Flyer/Slapper" .........................................6

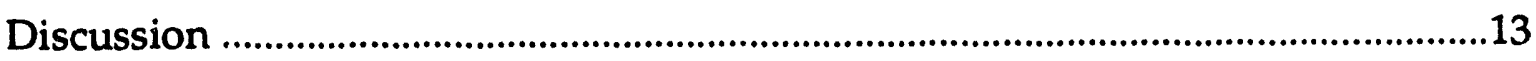

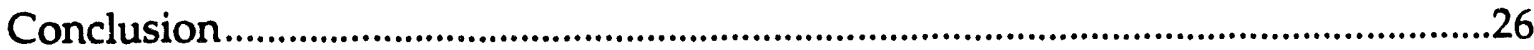

TASK 2: ACTUATOR-CABLE EXPLOSION IN CONTACT WITH HOT IHE .............29

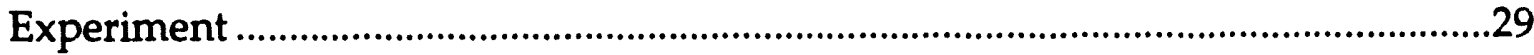

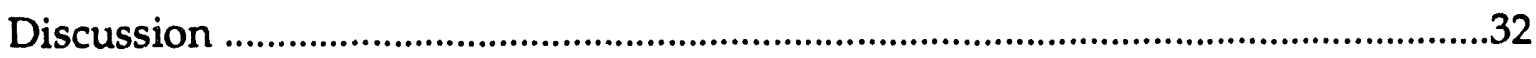

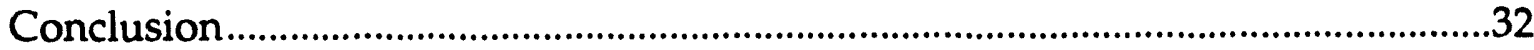

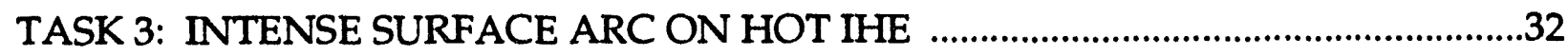

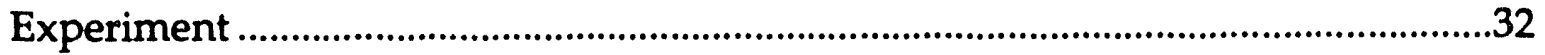

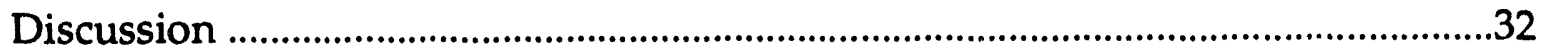

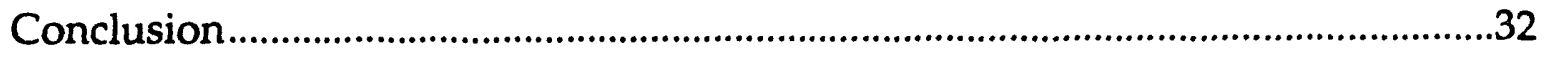

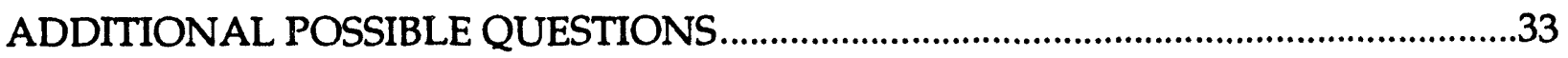

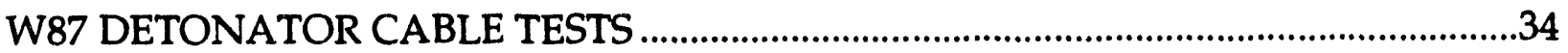

W87 Experimental Flyer/Slapper Velocity Measurement ......................................34

Flyer/Slapper Impact Characteristics ....................................................................37

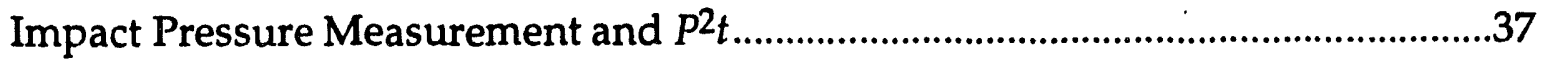

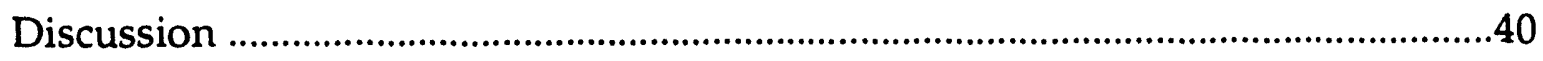

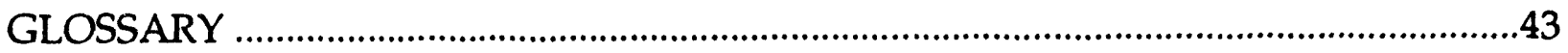




\section{FIGURES}

Figure 1. Schematic diagram of the arrangement of a section of detonator cable .7 on a disposable laminate for tests using our 87-kJ electric gun bank. The details of initial connections to the "top," "bottom," or "both" conductors of the flat twin-lead cable are shown.

Figure 2. Cross-section view of the twin-lead W89 cable with details of the two .8 copper leads and associated layers of Kapton insulation and adhesive.

Figure 3. View of the variable-length test fixture used for the $1.5-\mathrm{cm}$-wide detonator cable tests. The setup for a $2.54-\mathrm{cm}-(1-\mathrm{in}$.) long segment is shown after a shot was fired (the insulation had to be replaced after each shot).

Figure 4. Plot for Shot 87K of the cover-layer "flyer" velocity history for cable .11 explosions energized at a level of $31 \mathrm{~kJ}(60 \mathrm{kV})$ for examples of curves for the three different initial lead connections to $10.16-\mathrm{cm}$ - (4-in.) long test sections of W89 detonator cable.

Figure 5. Plot of the flyer cover layer velocity at a 2-mm standoff for "top," .13 "bottom," and "both" connections vs test cable section length.

Figure 6. Plot of the flyer cover layer velocity at a 7-mm standoff for "top," .14 "bottom," and "both" connections vs test cable section length. Also shown are the threshold velocities for initiation of ambient or hot LX-17, or ambient or hot Ultrafine TATB measured in Ref. 5 using 0.025-mm-thick Kapton flyer material.

Figure 7. Plot of the peak current vs length for a fixed voltage of $60 \mathrm{kV}$ for this .15 test series.

Figure 8. Arrangement for measuring the interface (particle) velocity to calculate the pressure just inside a thin gold or aluminum face LiF target from the known equation of state (EOS) of LiF.

Figure 9. The pressure history and time-integrated pressure (impulse or .18 momentum per unit area) for "bottom" lead initial connection test shot 87K051.

Figure 10. For Shot 87K051, the pressure-squared history (pressure shown in .19 Fig. 9) and the integrated value of $P^{2}$ over time are shown as an estimate of $P^{2 t}$ (note: the reflected wave and edge effects in the LiF crystal invalidate the late-pressure signal, so the final value used was taken at $0.1 \mu$ s after impact). 
Figure 11. For Shot 87K091, an example of an anomalously long pressure pulse ........20 from shot \#91 "bottom"-only irutial connection. The expected pulse duration for a $0.076-\mathrm{mm}-(0.003-\mathrm{in}$.) thick flyer at the measured velocity is about $13 \mathrm{~ns}$. The test series to resolve this unexpectedly long pulse is discussed in the text that follows.

Figure 12. Waveform plots for Shot $87 \mathrm{~K} 090$ on the transmission line for the case .22 of a "bottom"-only initial connection. The "bottom" conductor burst can be seen at just before $1 \mu \mathrm{s}$ from the $d I / d t$ and voltage signals, and the second main burst of the "top" conductor at about $2.0 \mu$ s launching the flyer.

Figure 13. Waveform plots for Shot $87 \mathrm{~K} 092$ on the transmission line for the .23 "both" initial connection case. The $d I / d t$ and voltage signals show the main burst of the two conductors at about $2.4 \mu$ s launching the flyer. The predictions of the CONFUSE code for peak current and peak current time, and for the burst current and burst current time, are also shown.

Figure 14. A streak picture record of the impact of the flyer strip on a glass .27 target placed $10 \mathrm{~mm}$ above the initial cable position. The case is for a "bottom"-only initial connection. Note the locations of the two longer duration features on each side. The streak writing speed was $100 \mathrm{~ns}$ per division.

Figure 15. A front and back surface view of the damage done to a 6.35 -mm-thick ......28 witness plate of aluminum located $10 \mathrm{~mm}$ above the detonator cable and impacted by the strip flyer shown in Figure 13. The enhanced damage grooves shown on each side of the strip centerline correspond exactly with the long duration features on the streak camera picture.

Figure 16. The arrangement used for go or no-go tests of the explosion of a 30 section of actuator cable embedded in a groove with ambient and $250^{\circ} \mathrm{C}$ pellets of LX-17 or UFTATB.

Figure 17. Cross-section exploded end view of the twin-lead W87 cable with .35 details of the two copper leads and associated layers of Kapton insulation and adhesive.

Figure 18. Waveform plots for Shot $87 \mathrm{~K} 119$ for the case of the "both" lead connection for a 10.16-cm (4-in.) length of W87 cable.

Figure 19. Plot of Shot 87K113 cover-layer "flyer" velocity history and integral 39 for a 10.16-cm (4-in.) section of W87 cable energized at a level of $31 \mathrm{KJ}(60 \mathrm{kV})$ for the case of "both" initial connection. The integral of the velocity shows the distance traveled as a function of time. 
Figure 20. For Shot 87K121, the pressure history and its integrated impulse for a .......41 "both" lead test case.

Figure 21. For Shot 87K121, the pressure-squared history (pressure shown in .42 Fig. 19) and its time-integrated value are shown as an estimate of $P^{2} t$ (value taken at about $0.1 \mu \mathrm{s}$ after impact, to avoid spurious late-time signals from edge reflections in the LiF crystal). 


\section{TABLES}

Table 1. Shots fired in first evaluation test ........................................................................10

Table 2. Shots fired in second evaluation task ..................................................................16

Table 3. Comparison of flyer velocity and impact data .......................................................25

Table 4. Actuator cable burst in contact with hot IHE (nonconfined) ...............................31

Table 5. Actuator cable burst in contact with hot IHE (confined).....................................31

Table 6. High-voltage discharges across hot IHE ...........................................................33

Table 7. Shot $\log$ of $\mathrm{W} 87$ detonator cable tests in $87-\mathrm{kJ}$ electric gun at $60 \mathrm{kV}$................38 


\section{EXECUTIVE SUMMARY}

This study was performed to explore and assess the worst-case response of a W89-type weapons system, damaged so as to expose detonator and/or detonator safing strong link (DSSL) cables to the most extreme, credible lightning-discharge environment. The test program used extremely high-current-level, fast-rise-time (1- to $2-\mu s)$ discharges to simulate lightning strikes to either the exposed detonator or DSSL cables.

- Discharges with peak currents above $700 \mathrm{kA}$ were required to explode test sections of detonator cable and launch a flyer fast enough potentially to detonate weapon high explosive (HE). With proper standoff between the cable and $\mathrm{HE}$, there is the possibility of launching a thin flyer with a velocity and local $P^{2} t$ exceeding the criterion for shock initiation of hot $\left(250^{\circ} \mathrm{C}\right)$ LX-17 and Ultrafine TATB (UFTATB) booster material and room-temperature UFTATB. The velocity was not sufficient, however, to initiate room-temperature $L X-17$.

- Detonator-safing-strong-link (DSSL) cables were exploded in direct contact with hot LX-17 and UFTATB. At maximum charging voltage, the discharge system associated with the HE firing chamber exploded the cables at more than $600-\mathrm{kA}$ peak current; however, neither LX-17 nor UFTATB detonated at $250^{\circ} \mathrm{C}$.

- Tests showed that intense surface arc discharges of more than $700 \mathrm{kA} / \mathrm{cm}$ in width across the surface of hot UFTATB [generally the more sensitive of the two insensitive high explosives (IHE)] could not initiate this hot IHE.

As an extension to this study, we applied the same technique to test sections of the much-narrower but thicker-cover-layer W87 detonator cable. These tests were performed at the same initial stored electrical energy as that used for the W89 study and hence with relatively higher energy/area deposited in the test cable.

- Because of the narrower cable conductor in the W87 cables, discharges greater than 550-kA peak current were sufficient to explode the cable and launch a fast flyer. This flyer generally had enough velocity to exceed the criterion for initiation of either room-temperature or hot LX-17 and UFTATB. However, the flyer/slapper is quite narrow (about $3 \mathrm{~mm}$ ) and may be much smaller than the critical spot size (at least at room temperature). Test information is needed on the critical initiation area (spot size) for narrow strips impacting room-temperature and hot IHE before the risk for initiation can be specified for the W87 detonator cables.

In summary, we found that lightning strikes to exposed DSSL cables cannot directly detonate LX-17 or UFTATB even at high temperatures, and they pose no HE safety threat. A similar statement can be made about a direct lightning strike to exposed HE. A lightning strike to an exposed detonator cable may explode the cable and launch the dielectric cover coat at high velocity. For a $700-\mathrm{kA}$ discharge (far in excess of $99 \%$ of all lightning strikes), the cover coat velocity from the W89-type cables 
has sufficient velocity (disregarding the critical initiation area) to initiate hot $L X-17$ and UFTATB and room-temperature UFTATB, but not room-temperature LX-17 To answer definitively the question of whether or not an exploding cable could initiate IHE, more experimental work needs to be done to measure the velocity thresholds for initiation at room and elevated temperatures and to measure the critical strip width for initiation. 


\section{INTRODUCIION}

A lightning strike is a natural source of high-power electrical energy that has a reasonable probability of energizing either the detonator or actuator conducting cables of a weapon that has undergone an accident, exposing those cables. The median strike would have a peak current of $20 \mathrm{kA}$ (kiloampere), but a positive stroke peak current in the $1 \%$ range could be $500 \mathrm{kA} .1$ Such currents can obviously melt or vaporize metal conductors with variable consequences depending on the peak current, rise time, geometry, and resistance of the current path. Lightning energy has been an obvious threat for abnormal environment weapon responses, and tests have been done on several weapon systems to demonstrate their invulnerability to typical peak currents. The W84 and W87 initiation subsystems have been studied and the results reported in a series of memos. ${ }^{2}$ Even lightning discharge currents of only a few kiloamperes through the detonator cable leads would likely explode the the tiny bridge foil in the detonator. However, unless the mechanical safing and arming device (MSAD) were in the armed position, the shock from this would be well shielded from the IHE components and would result only in dudding of the detonator.* One concern for higher current discharges is that the flat conductors used to keep the inductance low in fast-rise slapper detonator systems could be heated sufficiently by the current in a lightning discharge to explode and launch a flat "flyer" that could then shock-initiate maincharge explosives to detonation if other conditions were appropriate. However, the widths of the W84 and W87 detonator cables are significantly less than the characteristic critical spot size for short-pulse initiation of LX-17 at room temperature, minimizing this type of hazard. Another potential safety worry is the possibility of sufficient lightning current passing through the actuator cable to cause an explosion of the metal actuator cables in direct contact with the IHE.

The above concerns apply and are increased for the W89, because the W89 detonator cables are significantly wider than the W84 and W87 cables, which would be conducive to initiation by the cable cover-coat layer (especially if the explosive were heated in a fire). The W89 cable width is approximately the failure diameter of LX-17 at room temperature. In addition, recent one-dimensional (1D) experiments with hot TATB-based PBX-9502 have indicated a significant increase in sensitivity to shock initiation as compared with the sensitivity expected from earlier tests. ${ }^{3}$

*See Glossary for definitions of technical terms.

\footnotetext{
1 R. T. Hasbrouck, Lightning-Understanding it and Protecting Systems from its Effects, Lawrence Livermore National Laboratory, UCRL-53925 (April 10, 1989).

2 Dale Shauer, Defense Technology Engineering Division, LLNL, private communications (1990).

3 J. Dallman and Jerry Wackerle, Temperature-Dependent Shock Initiation of TATB-Based High Explosives," Los Alamos National Laboratory, P. 322 in Proceedings of the Tenth International Detonation Symposium (1993).
} 


\section{PREVIOUS EXPERIMENTAL TESTING}

Previous work on the W84 and W87 initiation subsystems involved model calculations and small-scale IHE experiments using short sections of the detonator cables and actuator cables. In addition, a lightning test was performed on a large-scale inert system mock-up, which revealed the qualitative response of the system components and included quantitative electric current measurements in the cables for the specific parallel circuit connections used as a load.

In the small-scale experiments, $2.54-\mathrm{cm}$ - (1-in.) long, 3.175-mm- (1/8-in.) wide cables were energized up to peak currents of $1100 \mathrm{kA}$ both in contact with and sepa:ated by $1.016 \mathrm{~mm}$ (0.04-in.) standoff from LX-17 and UFTATB (at 1.8- and $1.4 \mathrm{-g} / \mathrm{cc}$ density) at room temperature. Some detonations were observed with UFTATB at standoff, but none with LX-17. With a $25.4-\mathrm{cm}(10-\mathrm{in}$.) length of cable, no detonations were observed. ${ }^{2}$

Also in the previous work, $2.54-\mathrm{cm}$ - (1-in.) long sections of steel actuator cable were exploded with peak currents up to $1100 \mathrm{kA}$ in contact with LX-17, UFTATB (at 1.8-g/cc density), LX-10, and PBX-9407 at room temperature. Only the PBX-9407 detonated with $960-\mathrm{kA}$ peak current in the conductor.

Concerns about the magnitude of the increased sensitivity of hot TATB (as compared with ambient-teinperature TATB) have recently been raised. Run-todetonation-distance tests on PBX-9502 at $250^{\circ} \mathrm{C}$ have indicated a sensitivity to sustained pulse initiation similar to $\mathrm{PBX}-9501$ at room temperature. ${ }^{3}$ However, Livermore tests at $250^{\circ} \mathrm{C}$ on LX-17 continue to indicate an increased sensitivity to initiation, but not as much as the Los Alamos tests. ${ }^{4}$ Work at the two laboratories to resolve this difference for TATB-based IHEs is continuing. Older data, based on thin-flyer, short-duration pulse experiments and used as our reference base here, show only a moderately increased sensitivity to initiation of hot LX-17 and UFTATB at $1.8 \mathrm{~g} / \mathrm{cc}$ density (as compared with ambient temperature). 5

\section{SCOPE OF THIS INVESTIGATION}

As a result of the above concerns and requests to characterize the W89 initiationsystem response to adverse electrical conditions, we embarked on a series of experiments designed to measure the behavior of the major electrical components of the system under worst-case conditions. No consideration was given to risk analysis or to

\footnotetext{
4 Paul Urtiew, T.M. Cook, J. L. Maienschein, and C. M. Tarver, "Shock Sensitivity of IHE at Elevated Temperatures," Lawrence Livermore National Laboratory, p. 528 in Proceedings of Tenth International Detonation Symposium (1993).

5 R. Scheloske, L. Green, and R. Weingart, Sensitivity of Triaminotrinitrobenzene (TATB) at Elevated Temperature, Lawrence Livermore National laboratory, UCID-1836 (1980).
} 
the probability of insults that could result in these responses except to point out very general statistics.

We identified three tasks at the start of this study:

1. Determine the response of hot $L X-17$ and hot UFTATB when impacted by a detonator cable-cover-layer flyer produced by the worst credible lightning-current strike.

2. Go or no-go to detonation tests with sections of steel actuator cable electrically energized to explode in direct contact with hot LX-17 and hot UFTATB. We then repeated these tests with the hot UFTATB confined by a ceramic material during the actuator-cable explosions.

3. Measure the go or no-go to detonation response of hot UFTATB to a highcurrent surface arc discharge. (We decided to perform tests only on UFTATB because it is generally more sensitive to shock initiation than is LX-17, and we had only a limited supply of $\mathrm{L} X-17$ pellets at the time.)

We approached the three tasks as follows:

Task 1: The approach we took to the first task was different from that used in previous tests. Instead of doing experiments with live HE, we measured velocity' histories of the cable-cover-layer flyers for various cable lengths and conditions; then, we related these data to previous thin-flyer, short-pulse, shock-initiation data, ${ }^{5}$ which we believe were reliable. In addition, we measured impact pressure and pulse duration for a selected set of experimental parameters to characterize further the impact of the cable material and to relate our results in absolute terms to previous experiments made with flyers of different thicknesses.

Task 2: We conducted the exploding actuator cable tests with $L X-17$ and UFTATB in a geometry similar to that used in previous tests, ${ }^{2}$ except that we heated the IHE to about $250^{\circ} \mathrm{C}$. We repeated the set of experiments using hot UFTATB in a more confined geometry.

Task 3: We conducted the intense surface arc discharge study using samples of UFTATB at about $250^{\circ} \mathrm{C}$. The go or no-go tests involved connecting the $87-\mathrm{kJ}$ gun bank, charged to $40 \mathrm{kV}$, to $1.27-\mathrm{cm}-(0.5-\mathrm{in}$.) wide electrodes with a $1.27-\mathrm{cm}(0.5-\mathrm{in}$.) gap mounted on the surface of the UFTATB pellets. 


\section{TASK 1: EXPERIMENT}

\section{Detonator-Cable Explosion Launch of "Flyer/Slapper"}

The first task was carried out in the $100-\mathrm{kV}, 87-\mathrm{kJ}$ Electric Gun facility to achieve a fast (1- to 10- $\mu$ s-rise-time) current pulse to simulate lightning. Sections of twin-lead detonator cable with lengths from $2.54 \mathrm{~cm}$ ( 1 in.) to $22.86 \mathrm{~cm}$ ( 9 in.) were used in the tests. These cable lengths were selected to include the most likely cable lengths that might be involved in a lightning discharge after severe damage exposing the detonatorcable input leads. Cable lengths longer than $10.16 \mathrm{~cm}(4 \mathrm{in}$.) to $15.24 \mathrm{~cm}$ (6 in.) are unlikely, because of the thin dielectric insulation and expected electrical breakdown to the adjacent case. The actual cable connections to the gun bank were made according to Fig. 1. Three of the possible initisl alectrical connections to the bank from the twoconductor cable are indicated in . . figure. We tested these initial circuit connections to provide the worst-case energy deposition in the conductors; we expected that the "top" connection case would yield the fastest cover-coat velocity. Figure 2 shows an exploded view of the cable assembly with the various layer thicknesses. Figure 3 shows a postshot photograph of the experimental setup on the 87-kJ gun system. 6

Table 1 lists the shots fired for the first fiyer-velocity-evaluation task, including the important shot parameters and measured velocities at two selected displacements (continuous-velocity records are available for velocities at other stand-off distances). Cable lengths were varied from $2.54 \mathrm{~cm}$ ( 1 in.) to $22.86 \mathrm{~cm}$ (9 in.), and all were fired at $60 \mathrm{kV}$ except for one series. The highest currents and the fastest flyers resulted from the 2.54-cm (1-in.) lengths, of course, but, as the length increased, the currents began to fall and the velocities to decrease as expected. A $22.86-\mathrm{cm}$ (9-in.) length was the longest tested and resulted in velocities much too slow to initiate explosives as demonstrated below.

We measured velocity histories of the cover-coat material launched from the discharge through the cables using Fabry-Perot interferometry. An example of three records obtained from experiments on 10.16-cm (4-in.) lengths of cable is shown in Fig. 4. The "battom" connection generally yielded the highest velocity and allowed the longest flyer velocity history trace. The "top" connection yielded slower flyers, and the Fabry-Perot return signal disappeared within a few millimeters after launch, indicating that these "flyers" were coming apart or becoming too diffuse to reflect enough light to produce fringes. We expected that the "top" connection situation would yield the highest velocity, because less mass/area would have to be accelerated. However, it appears that breakdown always occurs between the two conductors after explosion of the first conductor, causing the "bottom" initial connection case to be favored. The "both" connection case produced an average flyer velocity nearly as high as the "bottom"-only connection. The velocity histories for the other cable lengths also generally followed the same order with minor variations according to the test cable

6 J.E. Osher et al., "Operating Characteristics and Modeling of the LLNL 100-KV Electric Gun," IEEE Trans. on Plasma Science, 17 (1989). 


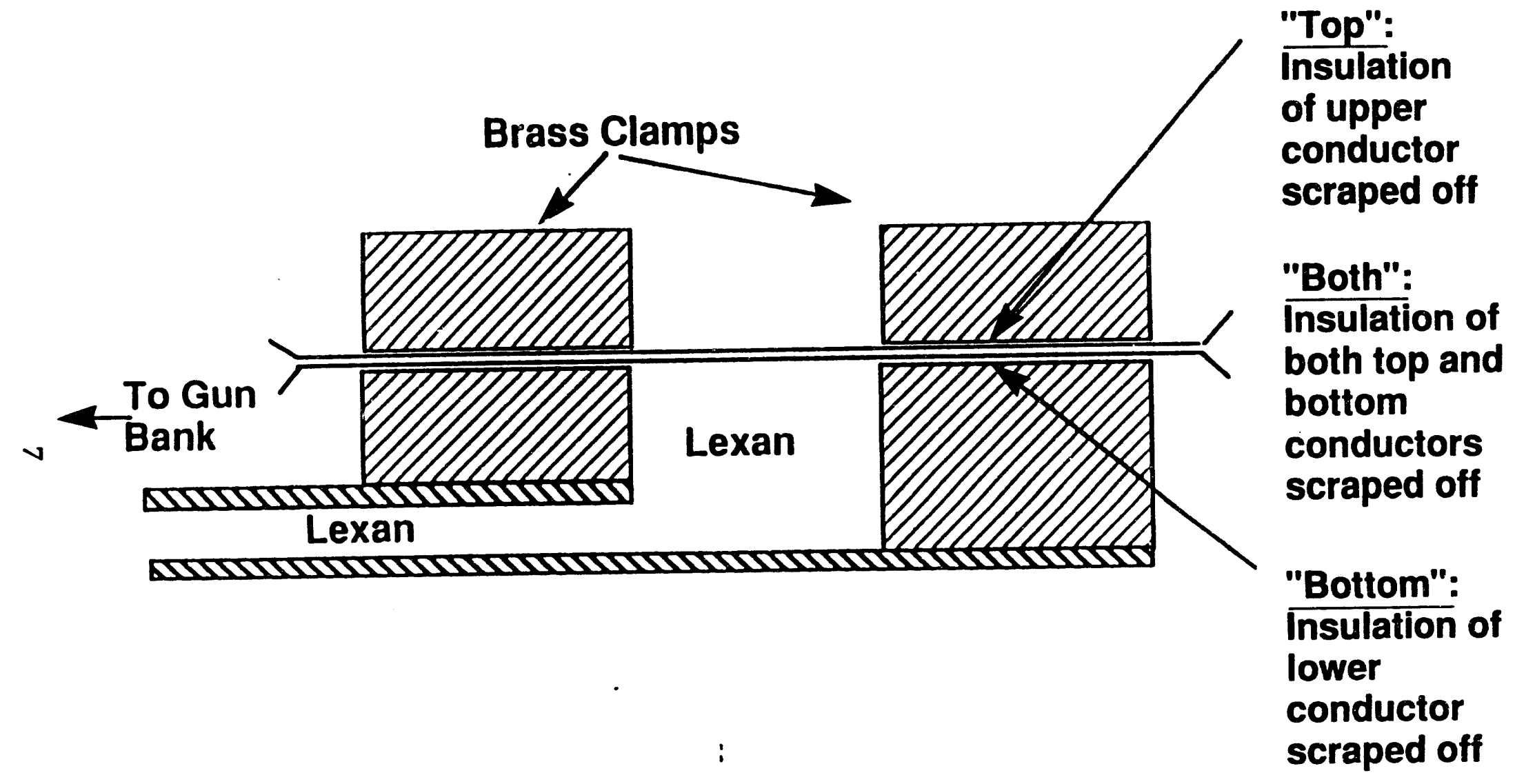

Figure 1. Schematic diagram of the arrangement of a section of detonator cable on a disposable laminate for tests using our 87-kJ electric gun bank. The details of initial connections to the "top," "bottom," or "both" conductors of the flat twin-lead cable are shown. 


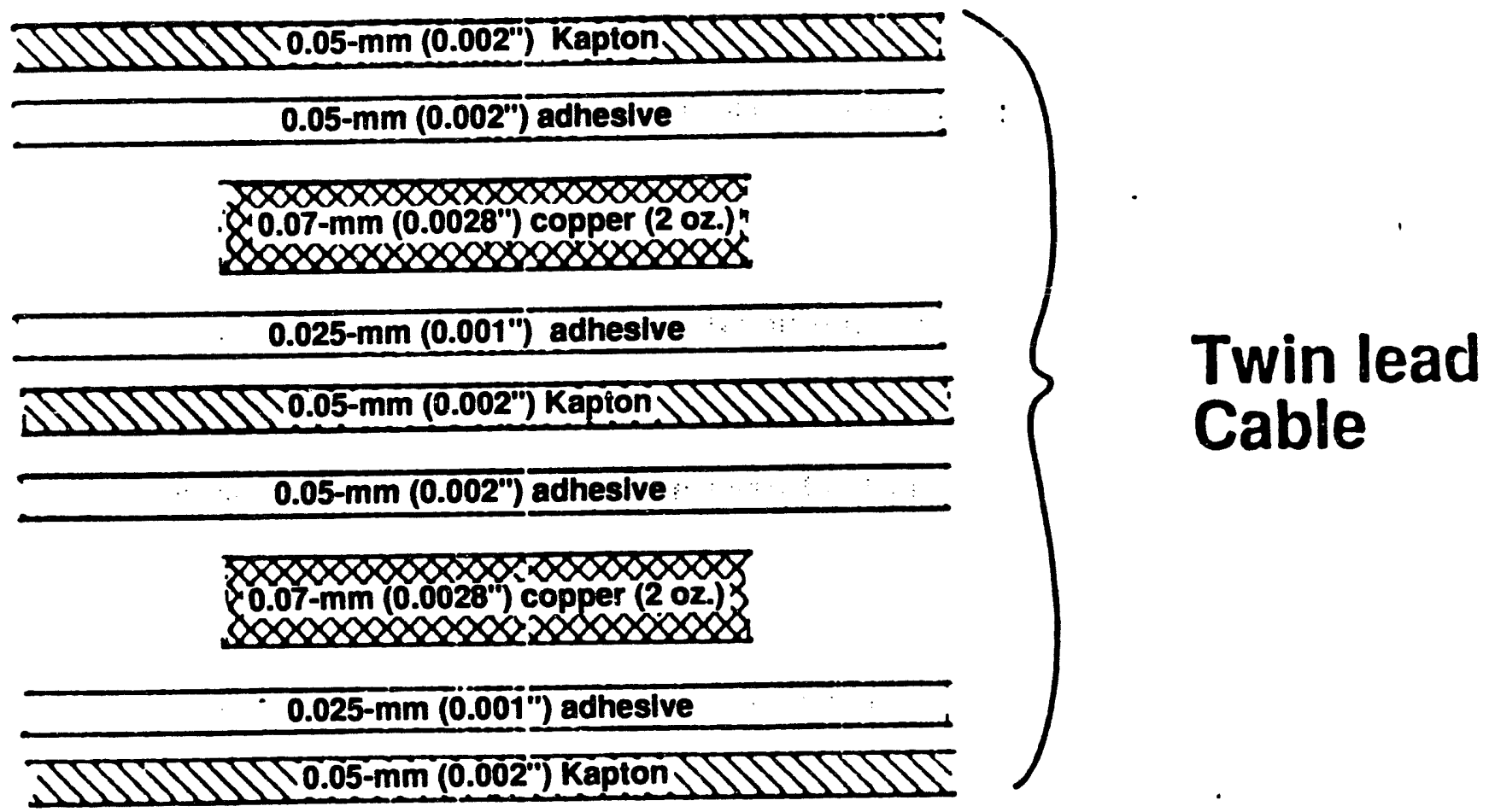

\section{Note: copper strip $\sim 1.5 \mathrm{~cm}$ wide}

Figure 2. Cross-section view of the twin-lead W89 cable with details of the two copper leads and associated layers of Kapton insulation and adhesive. 


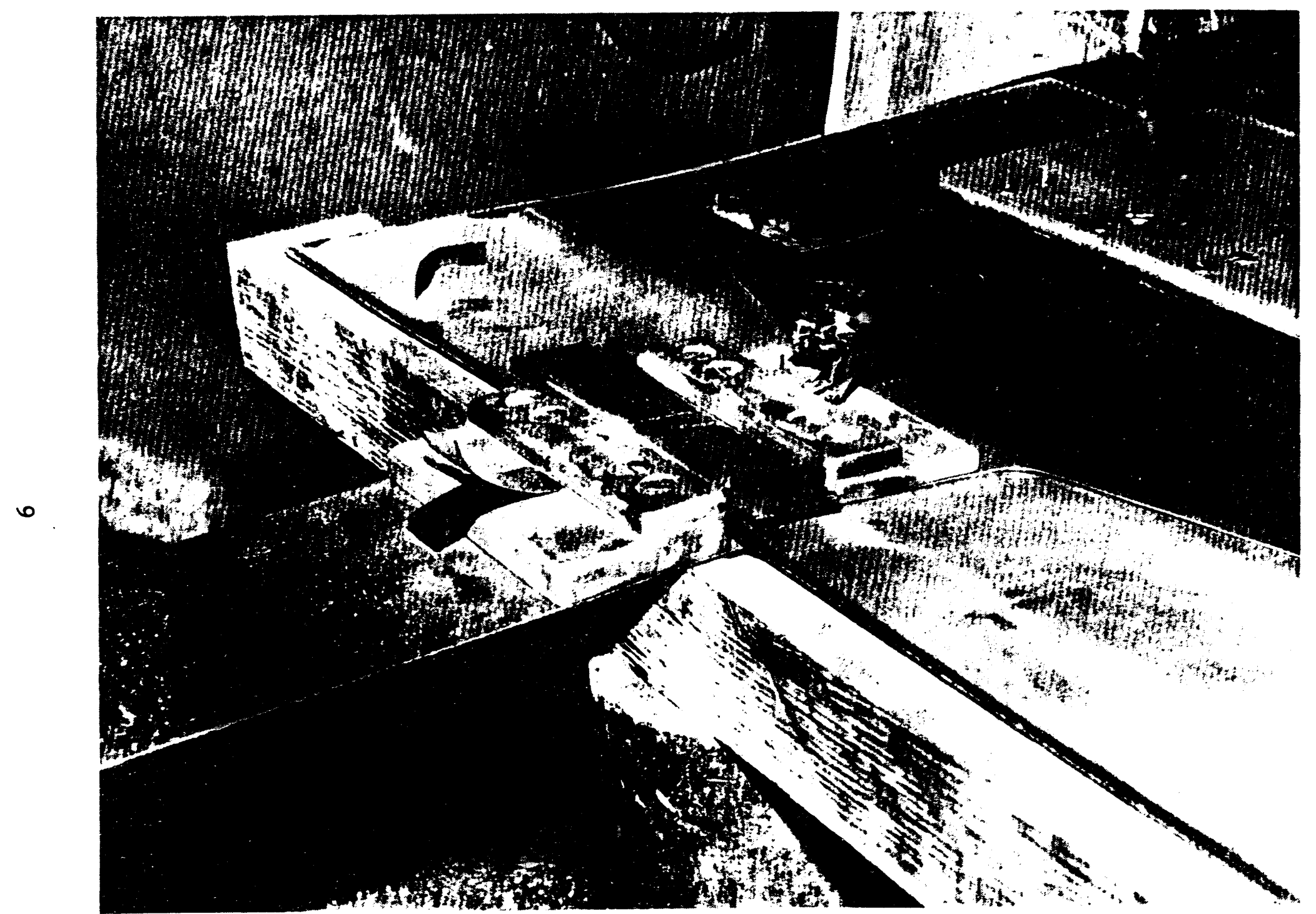

Figure 3. View of the variable-length test fixture used for the $1.5-\mathrm{cm}-$ wide detonator cable tests. The setup for a $2.54-\mathrm{cm}-(1-\mathrm{in}$.$) long segment is shown after a shot was fired (the insulation had to be replaced$ after each shot). 
Table 1. Shots fired in first evaluation test.

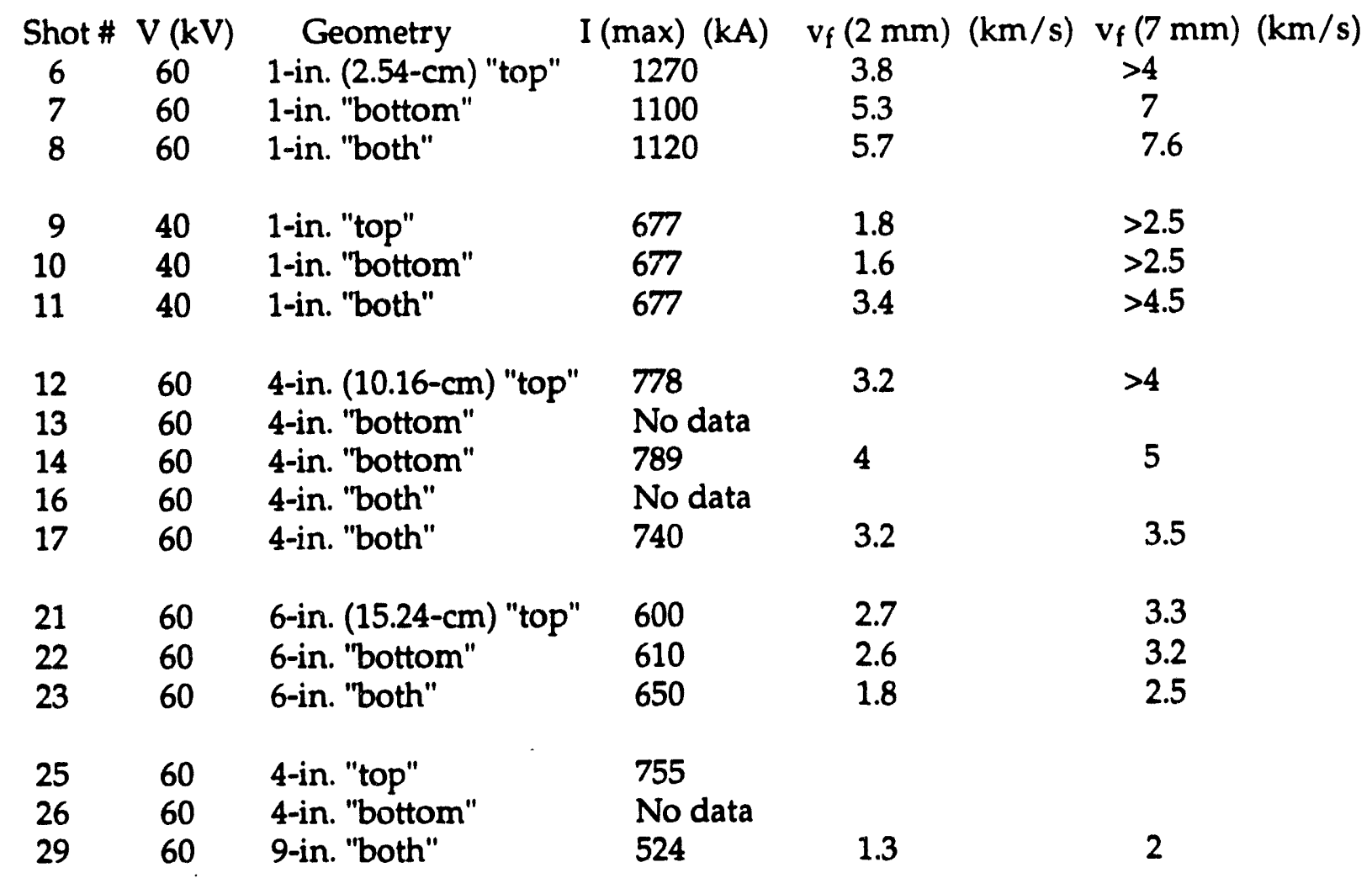




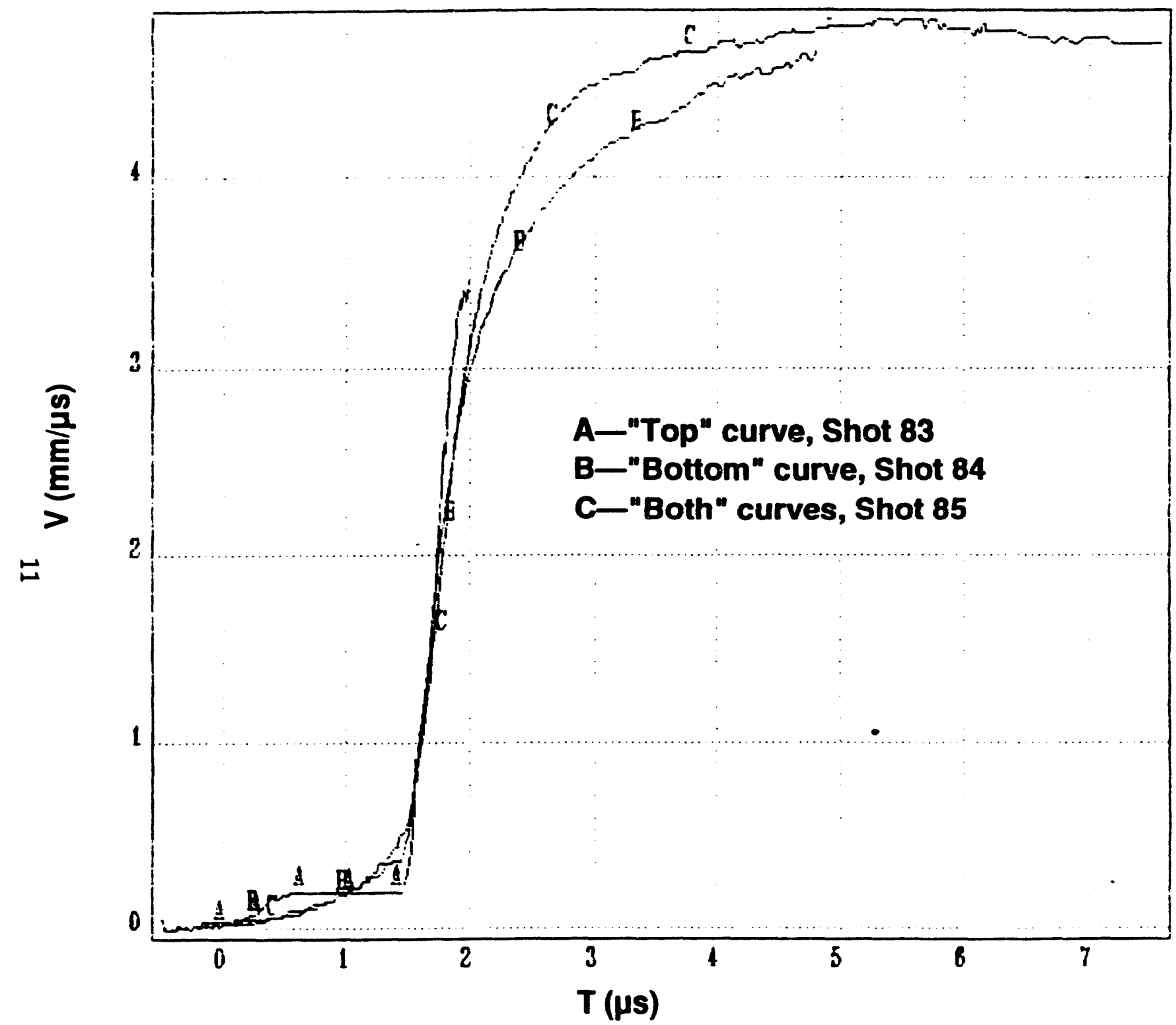

Figure 4. Plot for Shot 87K of the cover-layer "flyer" velocity history for cable explosions energized at a level of $31 \mathrm{~kJ}(60 \mathrm{kV})$ for examples of curves for the three different initial lead connections to $10.16-\mathrm{cm}-(4-i n$.$) long test sections of W89 detonator cable.$ 
length and the flyer distance traveled. Figures 5 and 6 show plots of the measured velocities for the various initial connections vs the length of cable at two stand-off distances, $2 \mathrm{~mm}$ and $7 \mathrm{~mm}$, respectively. Only minor velocity increases occur after $7 \mathrm{~mm}$, which is effectively an infinite distance.

Figure 7 shows the peak currents plotted vs the cable lengths for each connection case. The peak currents fall off with length (inductance), but not as rapidly as expected.

Because the initiation criterion for $\mathrm{HE}$ depends on the pressure, $P$, and the pulse duration, $t$, i.e., through some function of the form $P^{n} t$ with $n$ about 2.0, we designed a second series of experiments listed in Table 2 to measure the impact pressure and pulse width. Figure 8 shows details of the geometry used for our pressure-pulse experiments. Only 10.16-cm (4-in.) lengths were used with all three connection types. An impact block of clear lithium fluoride (LiF) was coated with a hydrodynamically thin coating ( $4000 \AA$ or less) of gold or aluminum to reflect the Fabry-Perot laser light and suspended $10 \mathrm{~mm}$ above the segment of detonator cable (at the middle). Figures, 9, 10, and 11 exhibit the results. The particle velocity of the $\mathrm{Al} / \mathrm{LiF}$ interface is measured and the pressure derived from the well-known LiF Hugoniot. Figure 9 shows an example of the pressure and the integral of the pressure (the impulse) plotted vs time for the "bottom" connection case. Figure 10 shows the pressure history and the integral of the pressure squared for this case. The results for the different connection cases are quite different. Only the "bottom" case seems to resemble a normal pulse expected from a simple single-layer planar flyer impact. However, the peak pressure value derived from the LiF measurement is less than that calculated for intact, full-density Kapton and the duration somewhat longer than that expected for the impact of a $0.076-\mathrm{mm}(0.003-$ in.) or 0.10-mm (0.004-in.) flyer. The other two ("top" and "both") initial connection cases resulted in lower-pressure, multiple impact pulses. Figure 11 shows the case for an anomalously long pressure pulse and resultant extreme value of $P 2 t$ (discussed below as one of several "unusual" shots with a "bottom"-only initial connection).

\section{Discussion}

Cover-layer velocity measurements: The first series of experiments was designed to evaluate conditions for launch of the cover layer as a flyer and to determine if this flyer has sufficient velocity to initiate ambient or hot LX-17 or UFTATB. The answer is clearly yes, particularly for lengths of $10.16 \mathrm{~cm}$ (4 in.) or less, based on the magnitude of the flyer velocity, as compared with the threshold velocity criteria in Ref. 5 of about $4.4 \mathrm{~km} / \mathrm{s}$ for ambient and $3.2 \mathrm{~km} / \mathrm{s}$ for $250^{\circ} \mathrm{C} \mathrm{LX}-17$, and about $2.8 \mathrm{~km} / \mathrm{s}$ for ambient and $2.3 \mathrm{~km} / \mathrm{s}$ for $250^{\circ} \mathrm{C}$ UFTATB (indicated as reference values in Figs. 4 and 5). Specifically, for the six shots with a 10.16-cm- (4-in.) long test section and detailed velocity records, all six shots had sufficient velocity at a standoff of $7 \mathrm{~mm}$ to exceed the velocity criterion to initiate either ambient or hot UFTATB or hot LX-17, but only four of the shots met the velocity criterion for initiating ambient LX-17. The necessary conditions include electrical connection to either exposed "bottom" or "both" conductors of the twin lead detonator cable and a microsecond-range rise time 


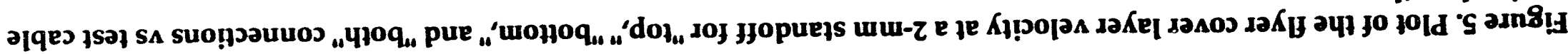

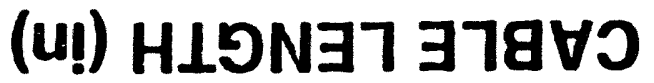

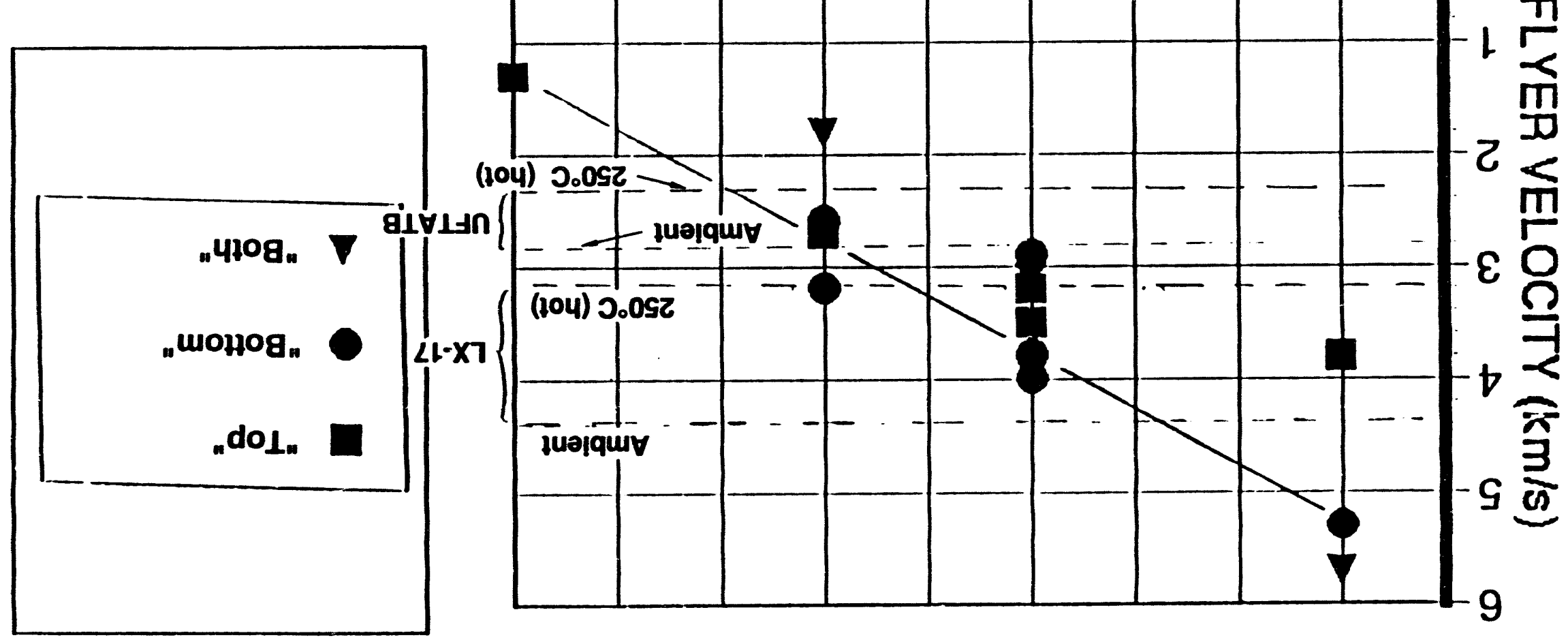




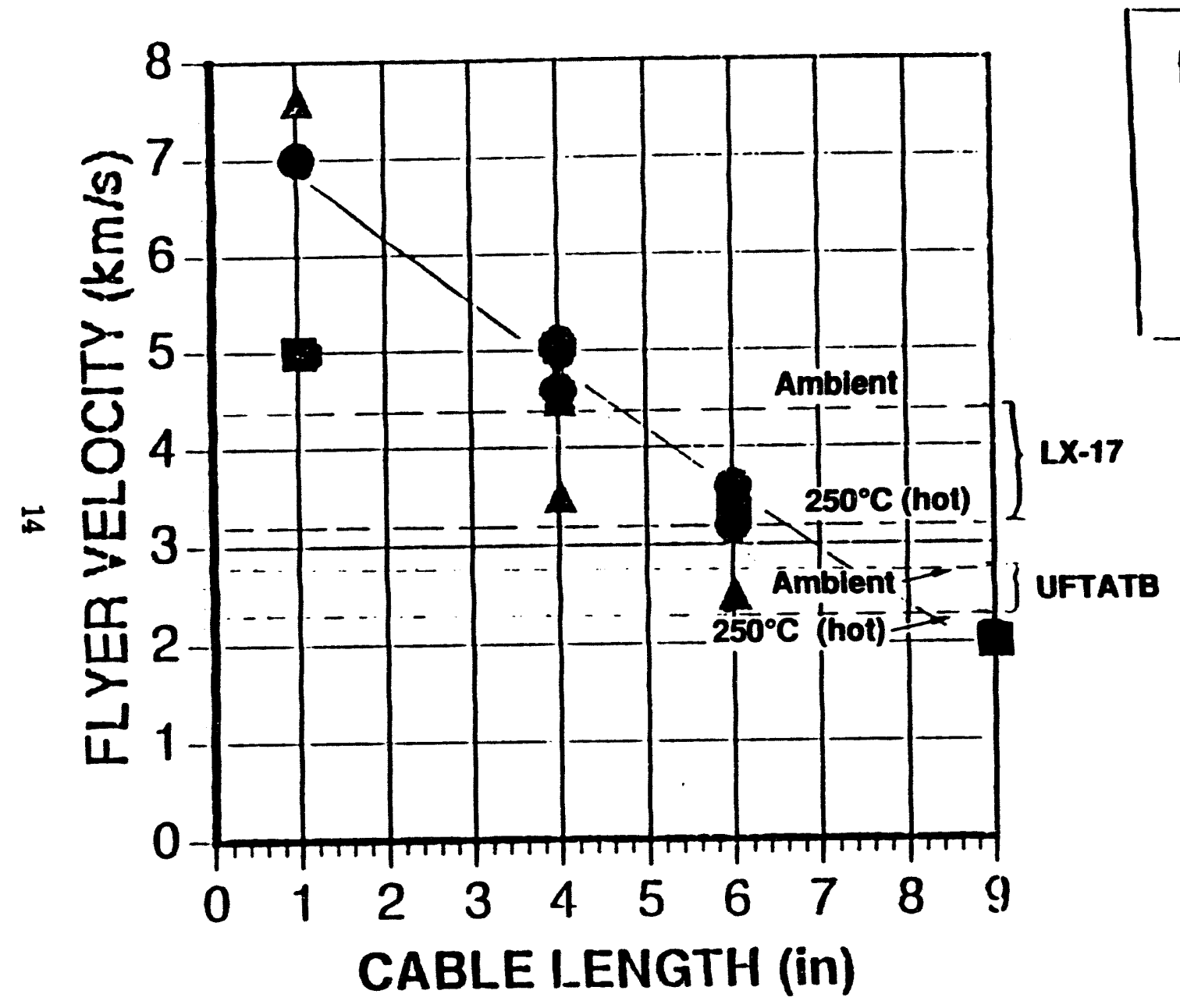

Figure 6. Plot of the flyer cover layer velocity at a 7-mm standoff for "top," "bottom," and "both" connections vs test cable section length. Also shown are the threshold velocities for initiation of ambient or hot LX-17, or ambient or hot Ultrafine TATB measured in Ref. 5 using 0.025-mm-thick Kapton flyer material. 


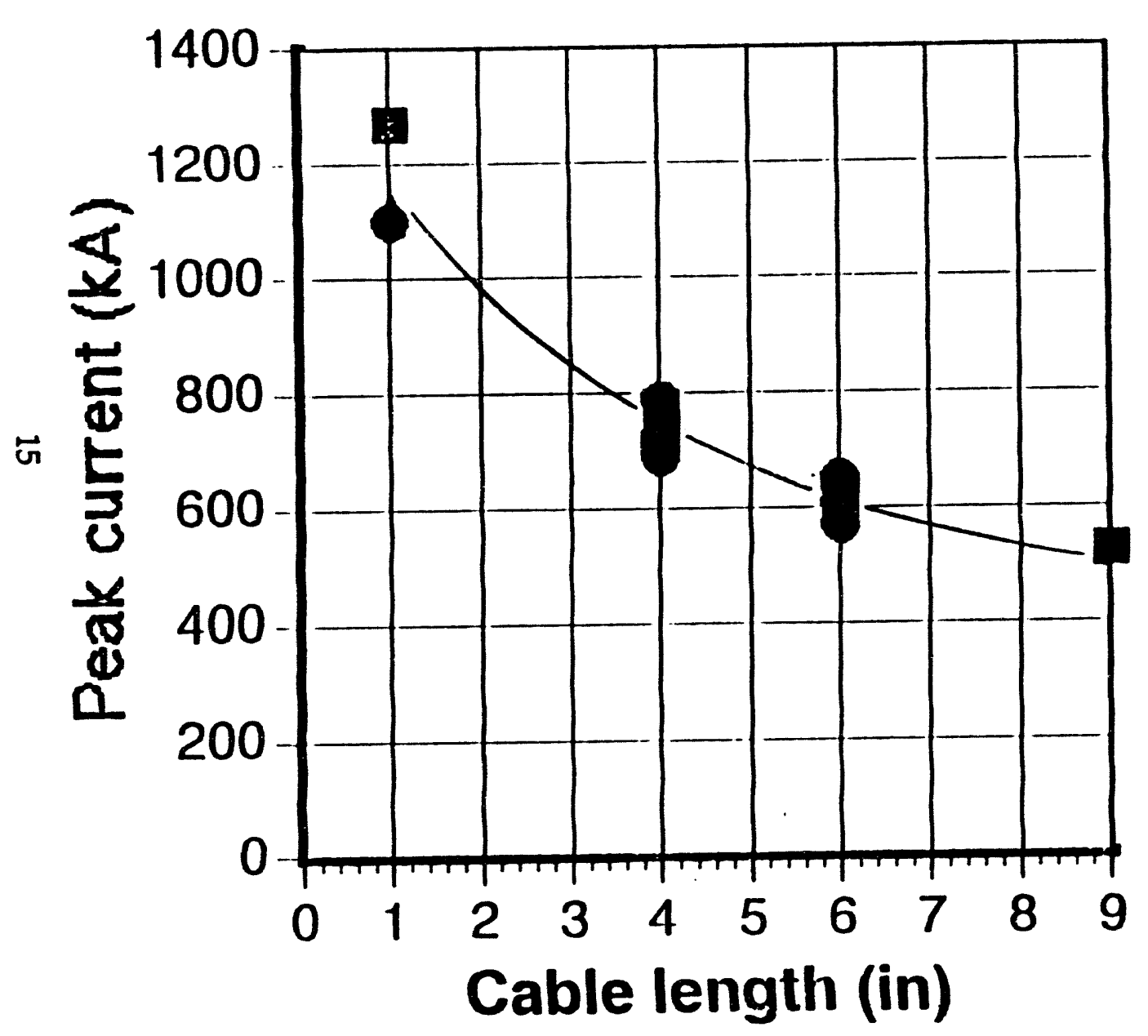

$\square$ "Top"

"Bottom"

$\Delta$ "Both"

Figure 7. Plot of the peak current vs length for a fixed voltage of $60 \mathrm{kV}$ for this test series. 


\section{$87 \mathrm{KJ}$ Electric Gun Log for NS shots 048-102 all at $60 \mathrm{KV}$}

Table 2. Shots fired in second evaluation task.

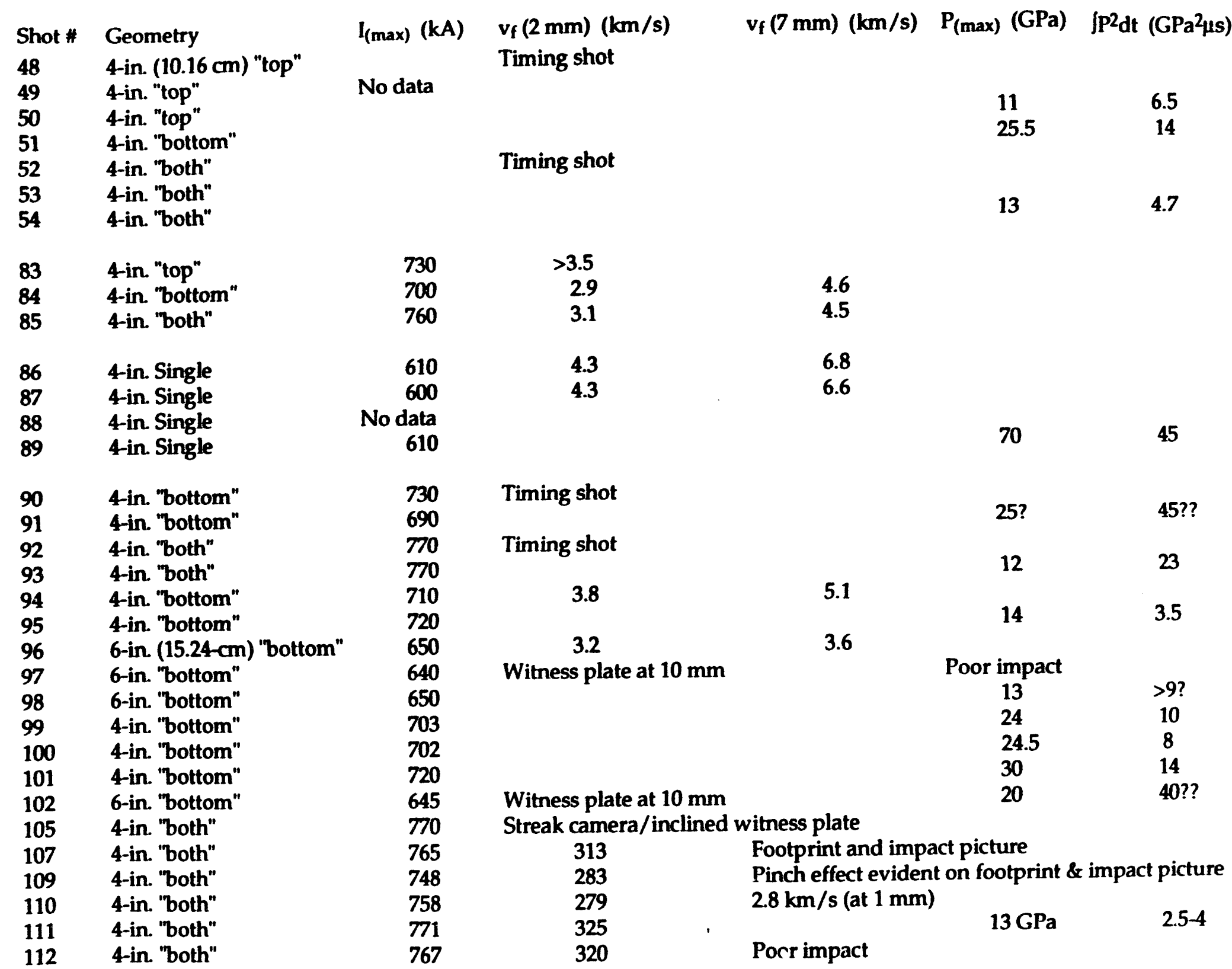




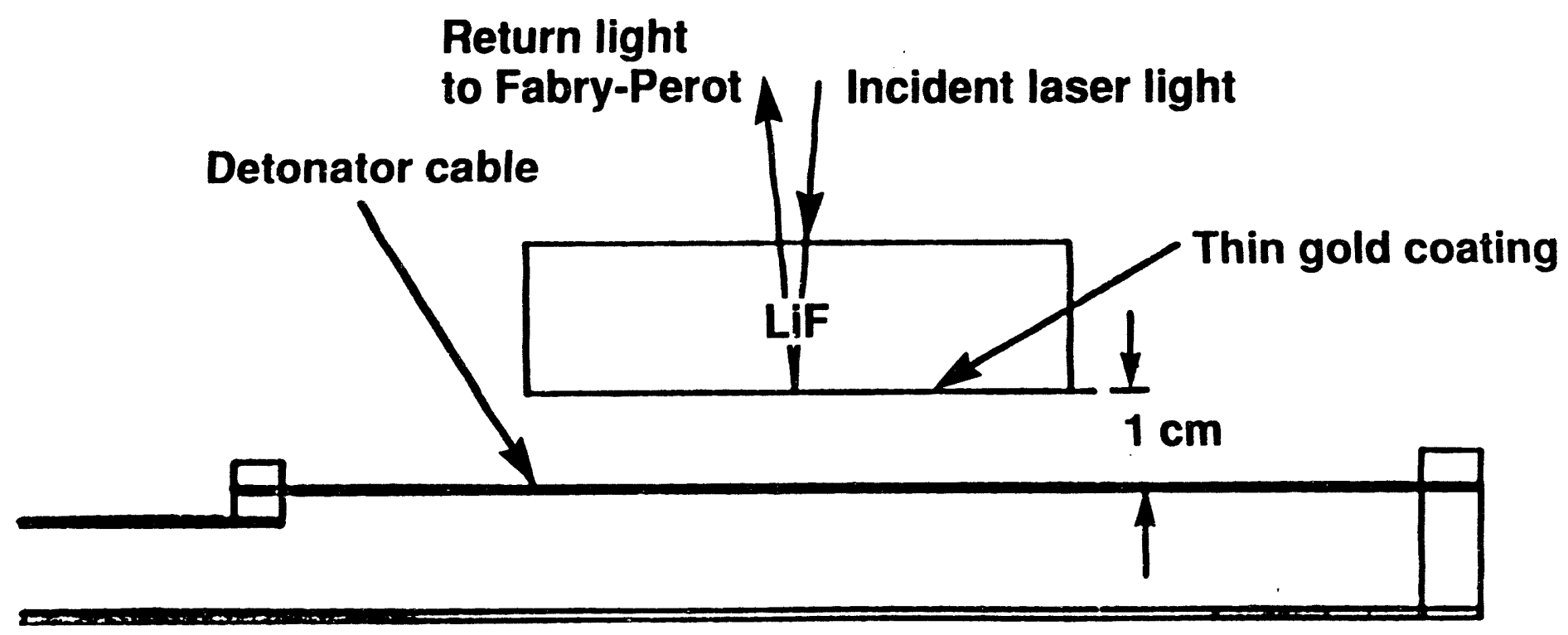

Figure 8. Arrangement for measuring the interface (particle) velocity to calculate the pressure just inside a thin gold or aluminum face LiF target from the known equation of state (EOS) of LiF. 


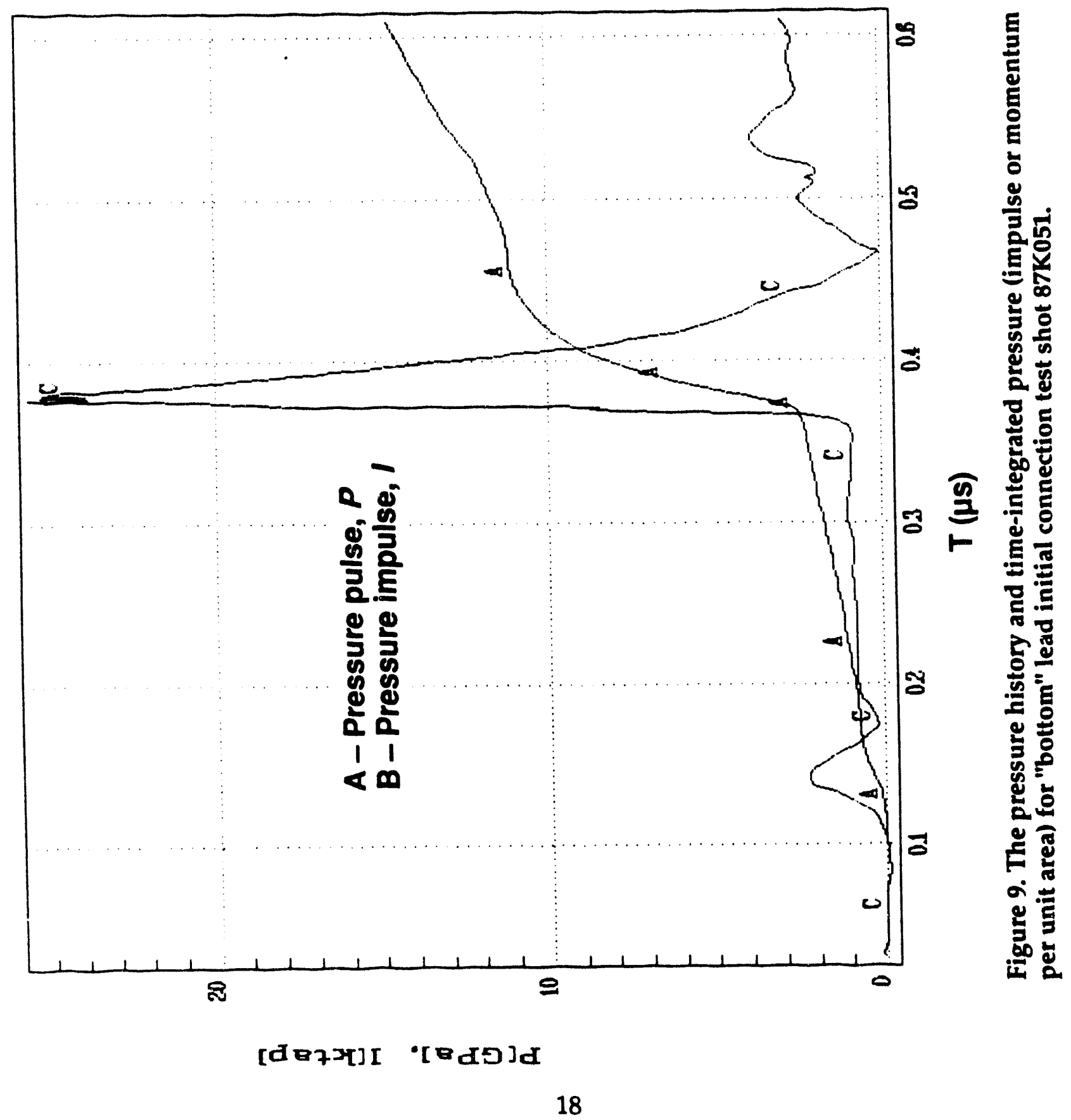




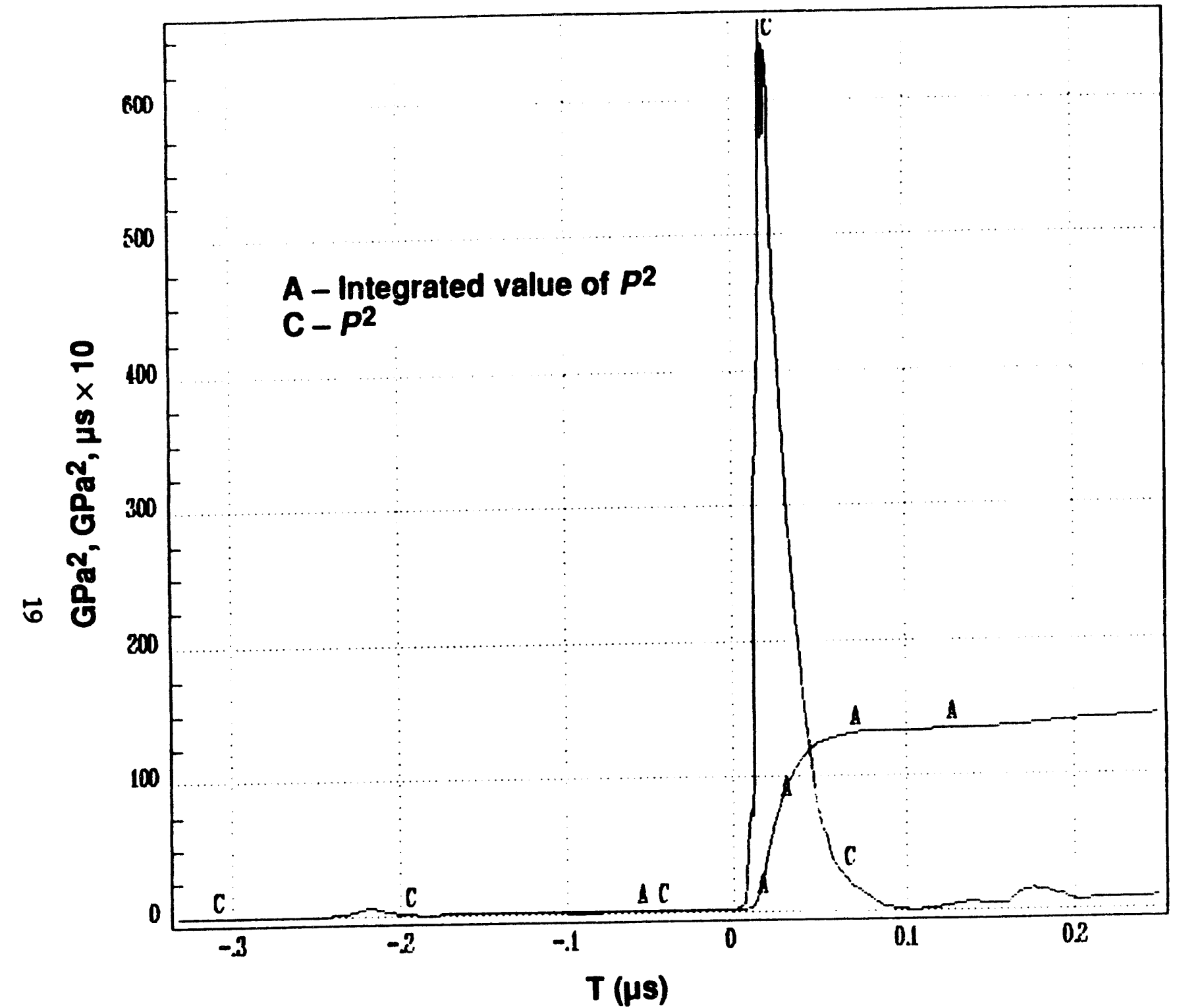

Figure 10. For Shot 87K051, the pressure-squared history (pressure shown in Fig. 9) and the integrated value of $P^{2}$ over time are shown as an estimate of $P^{2} t$ (note: the reflected wave and edge effects in the LiF crystal invalidate the late-pressure signal, so the final value used was taken at $0.1 \mu$ s after impact). 


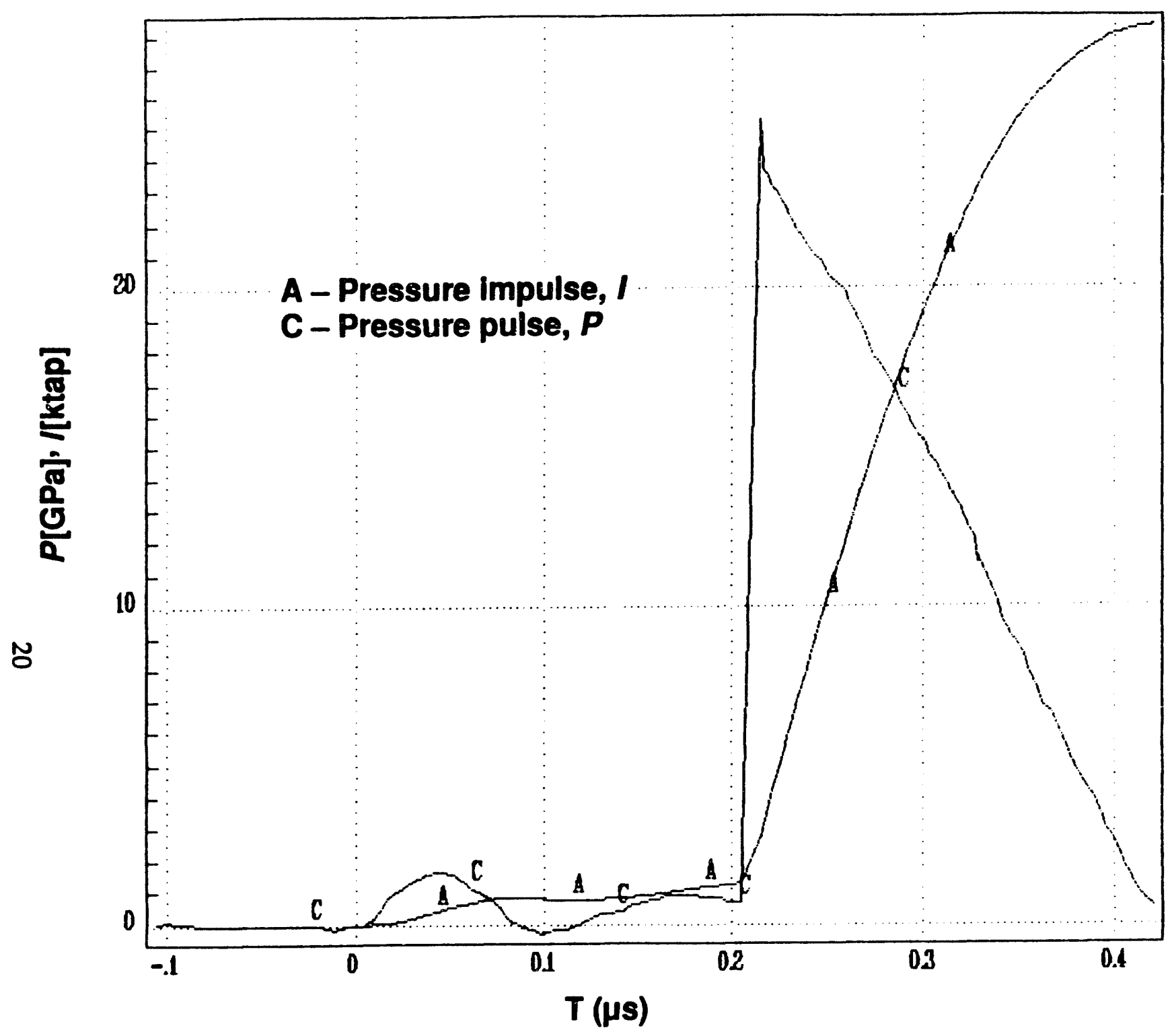

Figure 11. For Shot 87K091, an example of an anomalously long pressure pulse from shot \#91 "bottom"-only initial connection. The expected pulse duration for a 0.076mm- (0.003-in.) thick flyer at the measured velocity is about $13 \mathrm{~ns}$. The test series to resolve this unexpectedly long pulse is discussed in the text that follows. 
electrical discharge pulse producing a current in the cable of at least $700 \mathrm{kA}$. As noted in the earlier W87 study (Ref. 2), a lightning discharge to one or both of the cable leads and to the case as a common connection in parallel, with the return-circuit connection to the case at another point, would split the discharge current. Only a fraction of the total current (dependent on the specific circuit details) would then flow through the cable (interior to the case) and act to energize the explosion of the cable, with the rest of the current flowing through the case. The flyer velocities measured for cable lengths greater than about $15.24 \mathrm{~cm}$ (6 in.) are too low to be a hazard (at least for fast-rising test currents limited to about $700 \mathrm{kA}$ ). Only $1 \%$ of common negative lightning strokes exceed $200 \mathrm{kA}$, and only $1 \%$ of positive lightning (only $15 \%$ of global lightning) exceeds 400-500 kA, so the probability of slapper-like action is very low.

The record of the flyer velocity and its integrity varied more from shot to shot than was expected during the above tests. Detailed measurements of the voltage across the cable samples and $d I / d t$ (from the Rogowski belt signal) during the burst process show that for a single-conductor initial connection, the current to the first conductor was shunted to the second conductor within about $100 \mathrm{~ns}$ after burst. The sharp rise in resistance, and hence voltage, at burst of the first conductor apparently causes the insulation separating the two conductors to break down. The reduced voltage after breakdown and the shunting of the current to the second conductor sharply reduce the efficiency of the first conductor explosion for flyer acceleration. The second conductor then heats until it explodes, producing the main launch of the cover-layer flyer. To illustrate the burst sequence, the waveforms for the current $(I)$, the time rate of change of the current $(d I / d t)$, and the voltage for the "bottom"-only initial connection (shot \#90) for a 10.16-cm- (4-in.) long segment test are shown in Fig. 12. Note from the $d l / d t$ and voltage signals, the burst of first ("bottom") lead at about $1 \mu \mathrm{s}$. The burst of the second lead at about $2.0 \mu \mathrm{s}$ is really launching the flyer. For comparison, the waveforms for the current, the time rate of change of the current, and the voltage for Shot \#92 are shown in Fig. 13 for the "both" initial connection case with the main burst occurring at about 2.4 $\mu s$. For the single-initial-lead connection cases, this change in circuit during the burst process complicates the application of any code to predict the flyer data. However, for a "both" connection fixed circuit, the CONFUSE code7 (with the same total thickness bridge-foil) does a good job of predicting the peak current, the burst current, the timing of the burst, and the resultant flyer velocity history. The CONFUSE code predictions for the $I$ (peak) at the predicted peak time and $I$ (burst) at the predicted burst time are shown in Fig. 13. The measured flyer velocities for Shot \#85 at $2 \mathrm{~mm}$ and $7 \mathrm{~mm}$ are 3.9 and $4.6 \mathrm{~km} / \mathrm{s}$; the corresponding predicted values from CONFUSE are 3.9 and 4.9 $\mathrm{km} / \mathrm{s}$.

In the specific case of the "top" (initial) conductor connection, the current after the first explosion is shunted and inefficient for acceleration, and the second explosion is beneath the relatively heavy copper debris and interconductor insulation of the detonator cable, and hence is also relatively inefficient in launching the "top" thin Kapton cover to a high velocity. In the case of the "bottom" (initial) connection, the

7 I. Lindemuth, Los Alamos National Laboratory, private communication, and J. App. Phys. 57 (1958). 


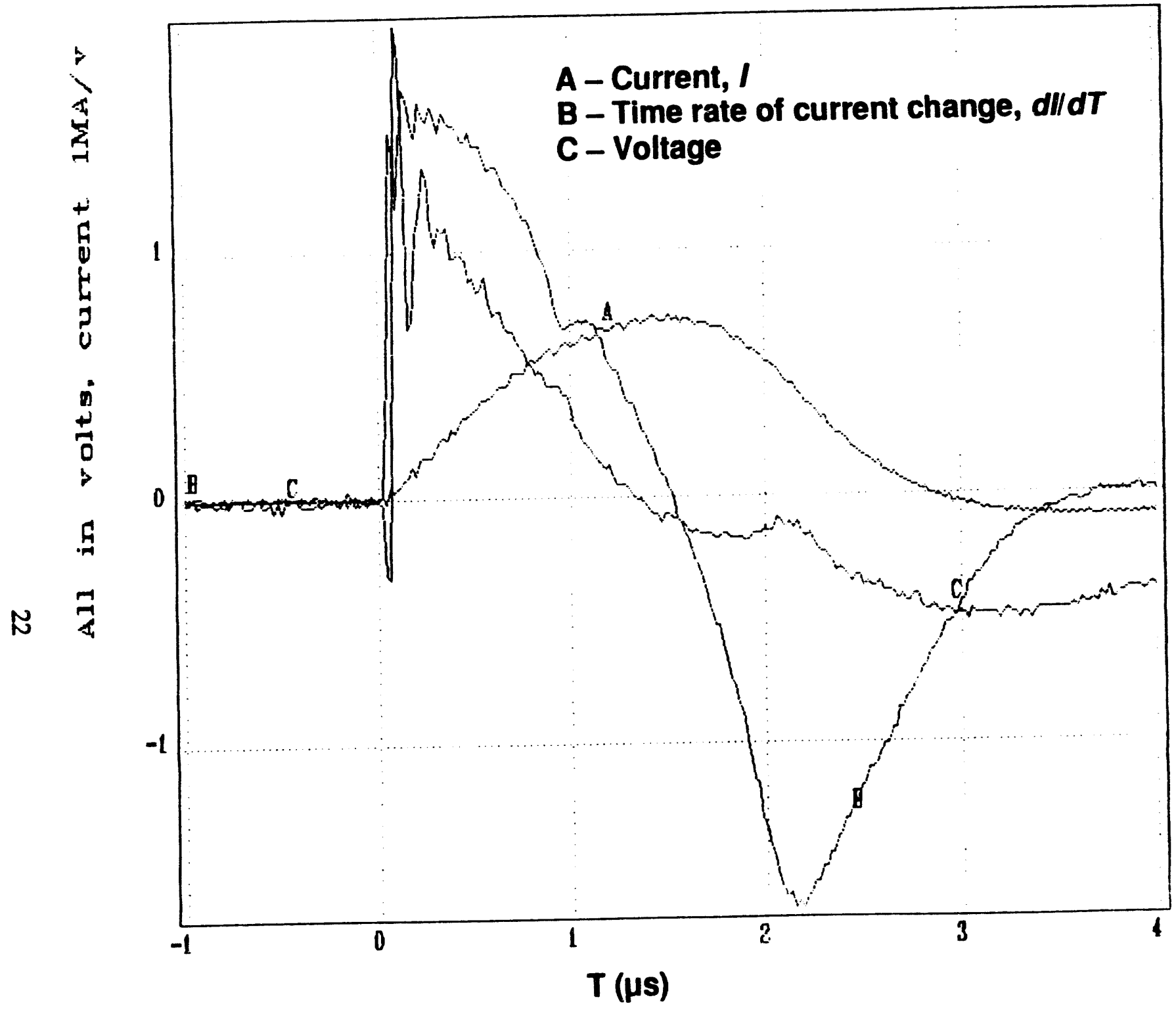

Figure 12. Waveform plots for Shot $87 \mathrm{~K} 090$ on the transmission line for the case of a "bottom"-only initial connection. The "bottom" conductor burst can be seen at just before $1 \mu \mathrm{s}$ from the $d I / d t$ and voltage signals, and the second main burst of the "top" conductor at about $2.0 \mu \mathrm{s}$ launching the flyer. 


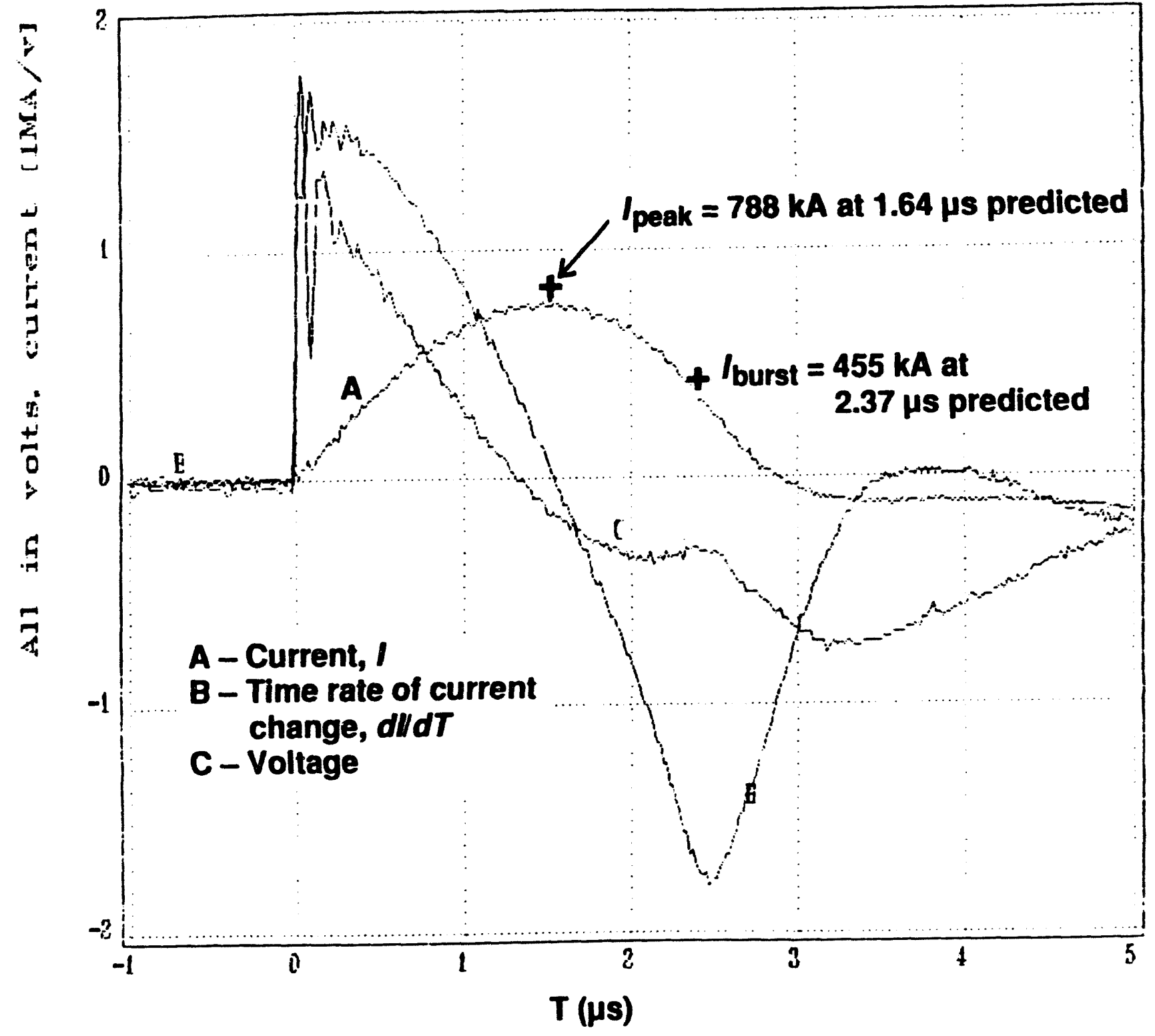

Figure 13. Waveform plots for Shot 87K092 on the transmission line for the "both" initial connection case. The $d I / d t$ and voltage signals show the main burst of the two conductors at about $2.4 \mu$ s launching the flyer. The predictions of the CONFUSE code for peak current and peak current time, and for the burst current and burst current time, are also shown. 
explosion of the first ("bottom") conductor favorably shapes the current pulse for the second "top" lead and produces a minor preacceleration of the "top" layers. The boosted "top" conductor is covered only by the thin layer of $0.076-\mathrm{mm}-(0.003$-in.) thick Kapton and adhesive, and its explosion now is generally the most efficient case of all for acceleration of the cover flyer. However, the delay time and the geometric location of the insulation breakthrough to the second conductor are variable and appear to contribute to an unexpectedly large variation in the final flyer-layer velocity. The case of the "both" (initial) conductor connection is less variable and produces nearly as high a cover-layer flyer velocity as the "bottom"-only initial connection. Tests were also performed with the twin-conductor cable split into two single conductor cables. Current passed through a single lead (half cable) yielded the highest flyer velocity of all (tests listed in Table 2). However, the type of damage likely to result in this case is felt to be extremely unlikely.

Impact pressure measurement for comparing $\boldsymbol{P}^{2} t$ : Given that the flyer velocity threshold criteria for hot LX-17 and UFTATB could be exceeded under some conditions, we designed a second series of tests, listed in Table 2, to measure the pressure history generated by impact of the cover-layer flyer. Reference 5 used $0.254-\mathrm{mm}-(0.010$-in.) thick Kapton flyers in establishing the short-pulse velocity criteria used above and shown in Figs. 5 and 6. Our approach to correcting the criteria for differences from the expected very-short-pulse duration produced from impact of the $0.0762-\mathrm{mm}-(0.003-\mathrm{in}$.) thick Kapton cover-layer flyer for the W89 was to compare values of $P^{2} t$ (considered one of the best parameters for characterizing the threshold for HE or IHE detonation, though some feel that the impact energy density may be an even better means of comparison). To calculate the value of $P^{2 t}$ for the velocity threshold data of Ref. 5, we use the EOS and our impact codes for calculating the pressure $P$ and the duration $t$ of Kapton flyer impact on $\mathrm{Al} / \mathrm{LiF}$ targets for the $0.254-\mathrm{mm}(0.010-\mathrm{in}$.) flyer thickness and threshold velocities used in Ref. 5 . We then compared these calculated $P^{2} t$ threshold values with our measured values of $P^{2}$ (impact on $\mathrm{Al} / \mathrm{LiF}$ ) integrated over the first $0.1 \mu \mathrm{s}$ or so after impact (reflected waves can invalidate the later signal). The pulse histories observed from the impact of the detonator cable-cover layer flyer are generally very short and spiked and occasionally include secondary impact structures, so direct measurement of $P^{2}$ times a duration time from the raw data is difficult. The compromise we adopted was to use the numerical integral of $P^{2}$ over time using the measured pressure history. This approach was found to be consistent with prior simple nearly rectangular pulse data using $0.3048-\mathrm{mm}-(0.012$-in.) thick Kapton flyers launched from a larger area electric gun geometry with a barrel used to define the flyer dimensions. This approach to $P 2 t$ is, however, subject to relatively large error bars from uncertainties in the "spiked peak" detail of the pressure history.

The comparison of $\mathrm{P}^{2} \mathrm{t}$ for Kapton impacting on $\mathrm{Al} / \mathrm{LiF}$ (shown in Table 3) again leads to some ( 2 out of 10 test shots for hot LX-17, 4 out of 10 shots for hot UFTATB, and 2 out of 10 shots for ambient UFTATB) of the measured local values of $P^{2} t$ from the 
Table 3. Comparison of flyer velocity and impact data.

Four-in. $(10.16 \mathrm{~cm})$ detonator cable at $60 \mathrm{kV}$ - (target aluminum faced LiF)

\begin{tabular}{cccc} 
Connection & Velocity $(d=7 \mathrm{~mm}) \mathrm{mm} / \mu \mathrm{s}$ & $P_{\max }($ from Up) GPa & $\int \mathrm{P}^{2} \mathrm{dt}(\mathrm{GPa}){ }^{2} \mu \mathrm{s}$ \\
\hline "Top" & 3.5 to $>4$ & 11 & 6.5 \\
"Both" & 3.5 to 4.5 & $13-25$ & $4.7-23$ \\
"Bottom" & 4.6 to 5.1 & $14-30$ & $3.5-14(45 ? ?)$ \\
\hline Single & & & \\
\hline
\end{tabular}

Previous shock criteria for 0.254-mm (0.010-in.) Kapton flyer on LX-17 (or UFTATB)

Temperature Threshold Velocity $P_{\text {calc }}$ (A1/LiF) $\tau$ (calc) $\quad \mathbf{P}^{2}$ (ont Al/LiF)

\begin{tabular}{ccccc}
\hline LX-17 & & & & \\
\hline Ambient & 4.4 & 28.5 & $58 \mathrm{~ns}$ & 47 \\
$250^{\circ} \mathrm{C}$ & 3.1 & 17.0 & $76 \mathrm{~ns}$ & 22 \\
& & & & \\
UFTATB & & 16 & $79 \mathrm{~ns}$ & 20 \\
\hline Ambient & 2.8 & 12 & $92 \mathrm{~ns}$ & 13 \\
$250^{\circ} \mathrm{C}$ & 2.3 & & &
\end{tabular}

detonator cable exceeding the corresponding threshold values calculated from Ref. 5. Note that we could have used the EOS for Kapton impact on LX-17 or UFTATB and calculated the $P^{2} t$ values for impact on these materials for the flyer velocities used in Ref. 5; however, we would then also have needed to convert our experimental pressure measurement on $\mathrm{Al} / \mathrm{LiF}$ to what we would have measured on impact with $\mathrm{LX}-17$ or UFTATB. As a result, we do not here give the $P 2 t$ threshold values for Kapton impact on LX-17 or UFTATB, although they can be calculated.

This pressure history study included some surprises. The impact of our cover layer of Kapton at a measured velocity on our $\mathrm{Al} / \mathrm{LiF}$ diagnostic target typically resulted in a sharply peaked pressure value about $10 \%$ below values predicted by our code calculations using the EOS for full-density Kapton (1.42), though the results can be fitted if the Kapton density is reduced to about 1 . In addition, the duration of the impact is generally observed to be longer than expected from impact of $0.0762-\mathrm{mm}$ (0.003-in.) Kapton at the measured velocity and occasionally significantly (up to nearly 10x) longer, as shown in Fig. 11.

Cover layer/flyer impact characteristics: In our third series of tests, we intended to evaluate the basic cause of the wide variation in our $\mathrm{P}_{t} t$ values. The results of these tests are also listed in Table 2. Tests with a $6.35-\mathrm{mm}-(0.25$-in.) thick witness plate of 
aluminum impacted at normal incidence at selected standoff distances from 1 to $10 \mathrm{~mm}$ (or at an inclined angle) from the detonator cable surface showed that the 15-mm-wide flyer (the initial width of the copper conductor) quickly narrows (presumably from magnetic pinch forces) to impact as only a 4- to 6-mm-wide strip after the first few mm travel after launch. Tests with a streaking camera observing the flyer impact on a glass target show the flyer to be still nearly flat at about $1 \mathrm{~mm}$, but rapidly narrowing and becoming more bowed (concave) with distance traveled. An example of the streak camera record of flyer impact on a glass target located at $10 \mathrm{~mm}$ is shown in Fig. 14 for a $10.16-\mathrm{cm}$ (4-in.) cable length with "bottom"-only initial connection. The witness plate damage from the impact of the "bottom"-only connection flyer, after $10 \mathrm{~mm}$ of travel, is shown in Fig. 15. The damage is dominated by two narrow deep grooves on each side of center. The dimensions and sfircing of the damage grooves correspond exactly to the side "fold" (longer duration) features seen in Fig. 14. In contrast, the damage pattern from the impact of a "both" connection flyer produces only one deep narrow groove on each side of center. Apparently, the time separation of the two explosions of the twinconductor detonator cable with an initial single-conductor connection produces a second edge convolution, as also seen on a streak image of the flyer impact on a glass target. If the duration of the light produced by impact is taken to be indicative of the pulse duration, these flyers involve a high-amplitude, short-duration pulse (tens of nanoseconds) on the flyer strip centerline, but include narrow side features 1 to $2 \mathrm{~mm}$ off the centerline at relatively similar pressures that can last longer than $100 \mathrm{~ns}$. If there is a $1.5-\mathrm{mm}$ misalignment in centering of the Fabry-Perot beam on the aluminum-coated LiF crystal mounted $10 \mathrm{~mm}$ above the cable (1-mm spot size typical) or if the flyer strip is launched at a slight angle, then the observed extreme variation in $P{ }^{2} t$ shown in Table 2 could occur. The composition of the side-fold features is not known; folding of the $15-\mathrm{mm}$ initial width strip as it is narrowed to about $5 \mathrm{~mm}$ is one possibility. Others are remnant debris from the tearing of the Kapton strip edge from the initial larger Kapton insulation sheet or even blowby of the high-pressure copper plasma accelerating the flyer material. In any case, the lowered peak impact pressure on the cints.rline (as compared with theory for full-density Kapton) is presumed to be caused by changes in the EOS of the Kapton as the strip is narrowed, deformed, and likely heated. The flyer velocity and impact data are compared with Ref. 5 in Table 3.

\section{Conclusion}

The flyer details suggest the need for repeated impact tests on $L X-17$ and UFTATB. The probability for the type of extreme abnormal event envisioned by Task 1 is clearly very low, though there may be some correlation for a damaging crash followed by a heating fire in a lightning-storm environment. The end result of the Task 1 test series is that this basic technique of measuring the "slapper"-like characteristics of the cover-layer strip flyer is inadequate to determine clearly the go or no-go criteria for ambient or hot LX-17 or UFTATB. The strip flyer layer produced by the electrical explosion of the cable conductors has been found to self-form a narrower and presumably thicker-than-expected flyer with deep folds and a wide range of local $P 2 t$ values. The results are suggestive of a possible extreme abnormal environment safety problem, but uncertainty about the sensitivity of LX-17 and UFTATB to critical spot size 
$i$ 


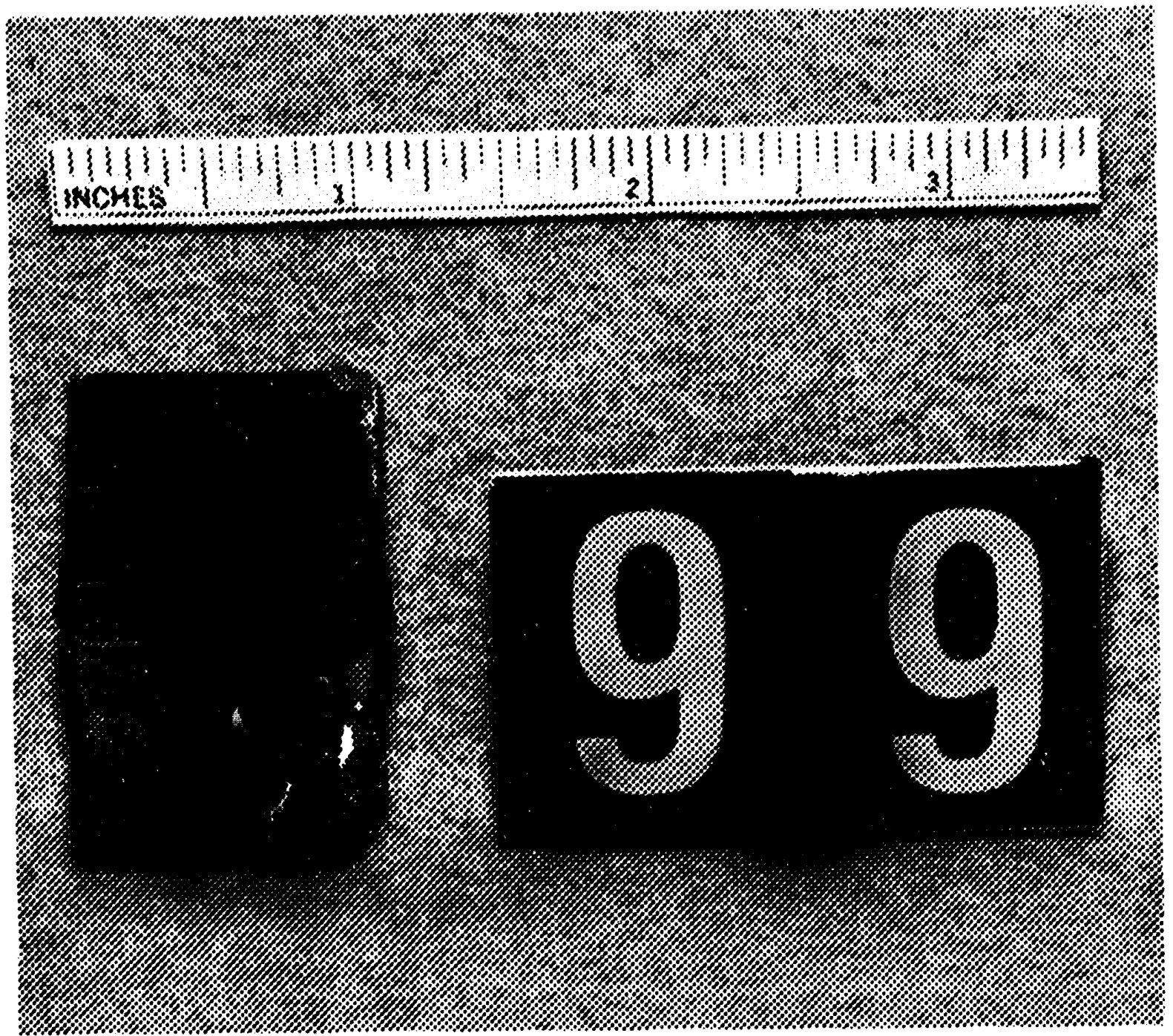

Figure 15. A front and back surface view of the damage done to a $6.35-\mathrm{mm}$-thick witness plate of aluminum located $10 \mathrm{~mm}$ above the detonator cable and impacted by the strip flyer shown in Figure 13. The enhanced damage grooves shown on each side of the strip centerline correspond exactly with the long duration features on the streak camera picture. 
features such as the nonuniform narrow strip flyer found here leads to significant uncertainty in applying the results. However, detonation of the main LX-17 charge over a 10.16- $\mathrm{cm}(4-\mathrm{in}$.) arc length could raise the question of some possible nuclear yield from a nominally one-point-safe system. The "bottom" line, in view of the extreme complexity of flyer impact size and detail, is that further selective go or no-go tests are recommended with both ambient and hot $L X-17$ and UFTATB exposed directly to the impact of the type of strip cover-layer flyer produced by electrical explosion of test lengths of detonator cable.

The velocity thresholds for flyer initiation of either ambient or hot UFTATB (1.8 density) are not discussed in detail here, other than to note that most of the test flyer impact conditions would be sufficient to initiate it. However, in a reasonably intact system, the UFTATB is protected from any exploding action of the detonator cable by a polycarbonate safety collar. Damage sufficient to dislocate this rugged collar located inside the case to expose the UFTATB would have to be extreme. However, at temperatures above about $155^{\circ} \mathrm{C}$, the polycarbonate would soften, melt, and perhaps run out of place, introducing the UFTATB as an additional possible hazard.

\section{TASK 2: ACTUATOR-CABLE EXPLOSION IN CONTACT WITH HOT IHE}

\section{Experiment}

This go or no-go test series involved the explosion of a short length of steel actuator cable in contact with either LX-17 or UFTATB at $250^{\circ} \mathrm{C}$. A $5.08-\mathrm{cm}-(2-\mathrm{in}$.) long section of cable was energized to ohmically heat and explode by connection to our 40-kV gun bank in HEAF. The IHE sample pellets were each $2.54 \mathrm{~cm}(1 \mathrm{in}$.) in diameter and $2.54 \mathrm{~cm}$ ( $1 \mathrm{in}$.) long, with a groove cut at one end so that the $3.175-\mathrm{mm}(0.125-\mathrm{in}$.) outside diameter actuator cable could be imbedded in it. A sketch of the setup is shown in Fig. 16. A heating mantle dome (destroyed in the course of each test) was used to raise the pellet temperature slowly to about $250^{\circ} \mathrm{C}$, as monitored by a set of thermocouples. The 5.08-cm- (2-in.) long section of actuator cable exploded at a current of about $480 \mathrm{kA}$ for the bank initially charged to $40 \mathrm{kV}$. Six tests were fired (three LX-17 and three UFTATB). Table 4 lists the details of the tests, all resulting in no-go conditions. A thin steel witness plate recessed to fit as a cap over one end of the IHE pellet was used as the go or no-go diagnostic.

After the tests were completed, we asked whether the results would have been different if the IHE pellets had been confined. We then performed a second brief test series using UFTATB (the more sensitive of the two IHE types used in these tests) at $250^{\circ} \mathrm{C}$ confined in a tight-fitting ceramic cup. We used a ceramic cup because it provided both a strong material for confinement and insulation to avoid shorting out the 40-kV bank connections. The cup was made of AD-995 ceramic (tensile strength of $40 \mathrm{ksi})$ with $5.08-\mathrm{mm}-(0.20$-in.) thick walls. We performed three tests using the hot UFTATB; again all resulted in no-go conditions. The test details are listed in Table 5. 

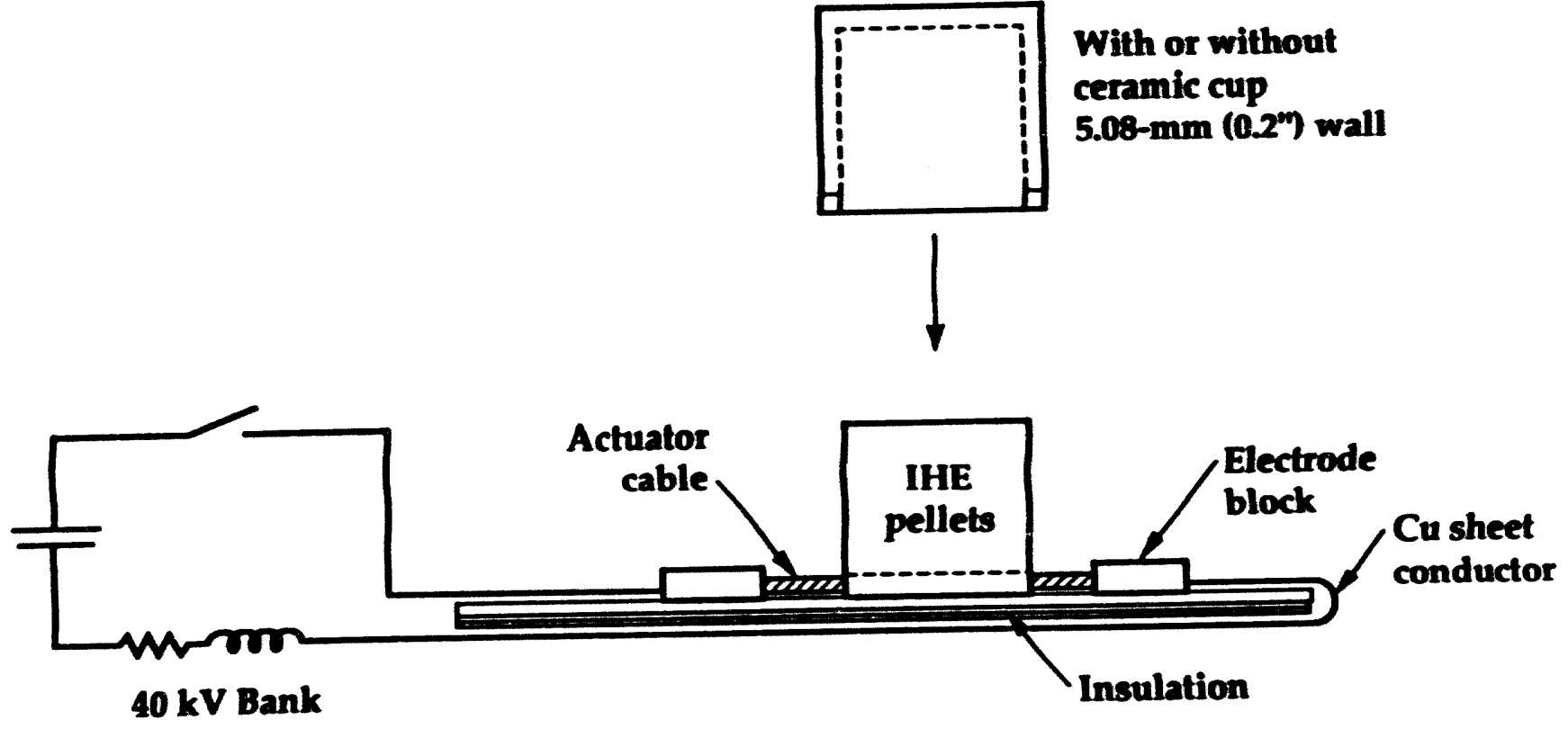

Figure 16. The arrangement used for go or no-go tests of the explosion of a section of actuator cable embedded in a groove with ambient and $250^{\circ} \mathrm{C}$ pellets of $\mathrm{LX}-17$ or UFTATB. 
Table 4. Actuator cable burst in contact with hot IHE (nonconfined).

- E-gun, $56 \mu \mathrm{F}$ at $40 \mathrm{kV}$

- Actuator cable, $5.08 \mathrm{~cm}$ ( 2 in.) long

\section{LX-17 (25 g)}

\begin{tabular}{ccccc} 
Shot \# & Temp $\left({ }^{\circ} \mathrm{C}\right)$ & Burst I (KA) & Peak I (KA) & Result \\
\hline NS2-4 & 256 & - & - & No go \\
NS2-5 & 258 & - & - & No go \\
NS2-6 & 249 & 486 & 627 & No go
\end{tabular}

UFTATB (25 g)

$\begin{array}{lllll}\text { NS2-7 } & 258 & - & - & \text { No go } \\ \text { NS2-8 } & 264 & 466 & 627 & \text { No go } \\ \text { NS2-9 } & 256 & 470 & 658 & \text { No go }\end{array}$

Table 5. Actuator cable burst in contact with hot IHE (confined).

- E-gun. $56 \mu \mathrm{F}$ at $40 \mathrm{kV}$

- UFTATB, $25 \mathrm{~g}$ confined in ceramic cup with 5.08-mm (0.200-in.) wall

- Actuator cable, $5.08 \mathrm{~cm}$ (2 in.) long

\begin{tabular}{ccccc} 
Shot \# & Temp $\left({ }^{\circ} \mathrm{C}\right)$ & Burst I (KA) & Peak I (KA) & Result \\
\hline NS2-10 & 250 & 457 & 615 & No go \\
NS2-11 & 254 & 405 & 598 & No go \\
NS2-12 & 272 & 407 & 582 & No go
\end{tabular}




\section{Discussion}

The tests performed represent the most extreme scenario expected from a direct lightning strike to a short length of exposed actuator cable in contact with hot LX-17 or UFTATB. In an actual situation, the W89 system would have to be damaged to expose the actuator cable. Further, the safety electrical shunt to the case at the cable opening would have to be damaged or destroyed, or most of the discharge current would be shunted externally to the case. A current level of more than $400 \mathrm{kA}$ is already below the $1 \%$ level for all lightning (positive and negative) discharges. All of the tests resulted in no-go, even using the violence of the actuator cable exploded at $480 \mathrm{kA}$ and a peak current in the cable of more than $600 \mathrm{kA}$.

\section{Conclusion}

The maximum credible lightning discharge to the actuator cable is not a safety problem to either hot UFTATB or LX-17.

\section{TASK 3: INTENSE SURFACE ARC ON HOT IHE}

\section{Experiment}

This series of tests was performed using our $40-\mathrm{kV}$ gun bank in HEAF charged to $40 \mathrm{kV}$ and connected across $1.27-\mathrm{cm}(0.5-\mathrm{in}$.) gap between two $1.27-\mathrm{cm}-(0.5$-in.) wide thin copper electrodes mounted in contact with the face of the hot IHE pellet. In an initial test of the setup with mock IHE at ambient temperature, framing camera pictures showed the discharge forming a multifilament discharge across the gap. Rogowski belt measurements showed a total peak current near $1 \mathrm{MA}$ across the $1.27-\mathrm{cm}-(0.5-\mathrm{in}$.) wide by $1.27-\mathrm{cm}-(0.5-$ in.) long gap. We performed three tests using UFTATB pellets (because they were more sensitive than $L X-17)$ heated to about $250^{\circ} \mathrm{C}$ using a heating mantle arrangement similar to that used in Task 2 . The main diagnostic to evaluate go or no-go was again use of a small steel plate cap recessed to fit over the top of the pellet. All of the tests resulted in no-go conditions with minor damage to the steel plate (detonation would have caused extreme damage) and collection of remnant UFTATB debris after each shot, as detailed in Table 6.

\section{Discussion}

This limited test series using UFTATB at $250^{\circ} \mathrm{C}$ as the most sensitive component resulted in only no-go conditions. The current density of the surface arc would appear to be at the limit expected from even the most extreme lightning.

\section{Conclusion}

An intense surface arc is not a hazard even for hot UFTATB. 
Table 6. High-voltage discharges across hot IHE.

- E-gun, $56 \mu \mathrm{F}$ at $40 \mathrm{kV}$

- IHE = UFTATB, 25-g pellet

- Arc gap $1.27 \mathrm{~cm}$ (0.5 in.)

\begin{tabular}{cccc} 
Shot \# & Temp $\left({ }^{\circ} \mathrm{C}\right)$ & Peak I (KA) & Result \\
\hline NS3-06 & 261 & 973 & No go \\
NS3-07 & 249 & 1,007 & No go \\
NS3-08 & 290 & 952 & No go
\end{tabular}

\section{ADDITIONAL POSSIBLE QUESTIONS}

The tests in the above set of tasks were all performed on full-density LX-17 or 1.8-density UFTATB at ambient and hot conditions (about $250^{\circ} \mathrm{C}$ ). We did not explore the question of the possibility of enhanced sensitivity of IHE cracked or partially rubbled to a lower density. If "damaged" IHE tests show a major increase in IHE sensitivity, some of the above tests should be repeated or even extended to include the possibility of case burn-through and surface arcing on such a damaged IHE sample. The main high-current pulse of positive lighting is quoted as being followed by a long discharge of several kiloampere range current lasting perhaps $150 \mathrm{~ms}$ and possibly causing case burn-through or significant weakening.

The tests for the above tasks were done using a relatively fast-rise (rise time near $2 \mu \mathrm{s})$ current pulse typical of negative lightning. The results of these tests indicate that positive lightning represents the more serious threat, because of the extremely highcurrent pulse required to explode the cable and launch a hazardous energetic flyer. Therefore, if the above strip impact tests on IHE show significant probabilities to "go to initiation," selected additional tests should be performed with much slower rise-time (10- to 50- $\mu \mathrm{s})$ pulses more typical of positive lightning. A preliminary calculation using the CONFUSE code indicates that this would have a significant effect in reducing the peak current needed for exploding the detonator cable, but would also produce a much lower velocity strip flyer. (For instance, even an 8- $\mu \mathrm{s}$ rise-time pulse would reduce the peak current to $520 \mathrm{kA}$, with a flyer velocity of $1.3 \mathrm{~km} / \mathrm{s}$ at $2 \mathrm{~mm}$, or just over $4 \mathrm{~km} / \mathrm{s}$ at $7 \mathrm{~mm}$ ).

In any case, this study did not explore the chain of probabilities in a real scenario resulting in a significant hazard. Briefly, such probabilities would include the event resulting in sufficient damage to expose the cables, the likelihood of a lightning 
discharge of sufficient current to launch an energetic flyer including the probability of the various discharge circuit connections (separate from or in parallel) to the case and return circuit splitting the current so that only a fraction flows through the cable, the effective interior cable length undergoing the explosion (limited in length by voltage breakdown to the adjacent case wall), and finally the interior cable to IHE local stand-off distance in the damaged system (a stand-off distance of several millimeters is required to get the flyer velocity up).

\section{W87 DETONATOR CABLE TESTS}

The W89 test series contributed considerable insight as to the type of slapper action that might result from a "lightning-like" electrically energized explosion of the W89 detonator cable. Therefore, we decided to perform a selected set of similar tests with the W87 cable using the same techniques to evaluate the margin of safety with that system. The W87 cable has much narrower copper conductors (3.175-mm or 0.125-in.-wide as compared with the 15-mm width for the W89) and a slightly thicker $(0.127-\mathrm{mm}$ or 0.005 -in. instead of the $0.076-\mathrm{mm}$ or 0.003 -in. for the W89) overcoat of Kapton. The bonded Kapton total width of the cable is about $6.35 \mathrm{~mm}$ (0.25-in.). The copper conductor thicknesses are the same for both systems.

\section{W87 Experimental Flyer/Slapper Velocity Measurement}

The flyer/slapper velocity measurement was again carried out using the 87-kJ electric gun facility to simulate a high-current lightning discharge. Four-in. $(10.16-\mathrm{cm})$ test lengths of cable were selected as representing the longest cable length (and hence most serious problem from the standpoint of initiating the IHE over an arc length rather than a single point) that would likely be involved in a lightning strike before electrical breakdown through the insulation to the case shunting the current from the cable to the case. Shorter lengths would be expected to yield still higher flyer/slapper velocities and longer lengths lower velocities, all within a relatively narrow range of required peak current levels. A cross-section view of the W87 cable is shown in Fig. 17. We selected the "both-leads" initial connection for test (with insulation scraped off from the ends of both the "top" and "bottom" conductors) as the most likely connection in a lightning strike to damaged leads and representative of the peak current needed and resulting in an average-to-maximum flyer/slapper velocity. The W89 tests showed that the two leads of the twin-lead cable eventually short together (from interlead dielectric breakdown when exposed to the high-voltage spike at burst of the first connection if the single-lead initial connection is selected), and current sufficient to burst both leads is required to launch an energetic flyer/slapper. (The flyer velocity attained with the "bottom"-only initial connection can be highest of all for the three different initial lead choices, but it is more variable in performance.)

We kept the charge voltage of the 87-kJ gun bank at $60 \mathrm{kV}$ (31 kJ) for comparison with the W89 test series (a somewhat arbitrary choice). A typical set of waveforms for I and $d l / d t$ are shown in Fig. 18. The measured peak current is about $550 \mathrm{kA}$, which can be 


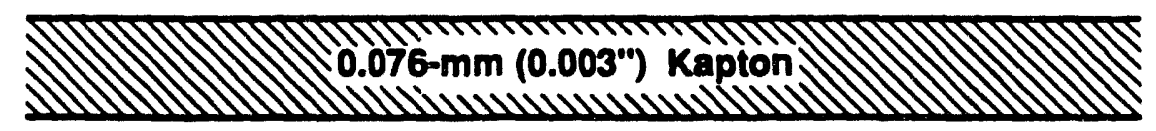

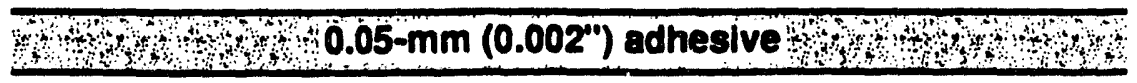

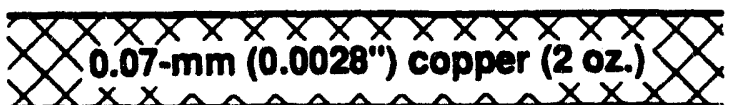

$\therefore \because \because \because 0.025-\mathrm{mm}(0.001 \mathrm{~W})$ Epoxy $\because \because \cdots \cdots$

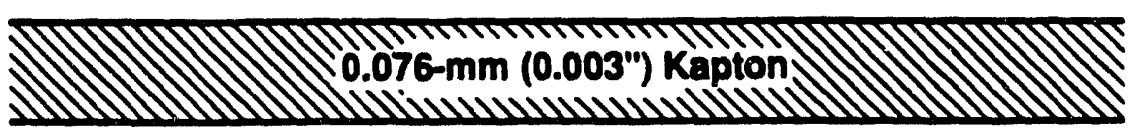

Twin lead cable

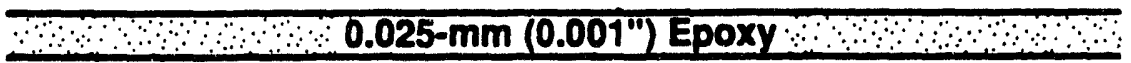

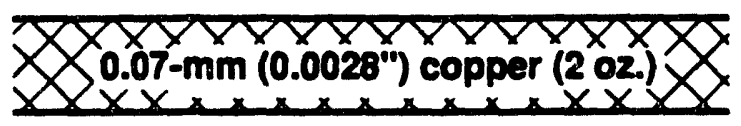

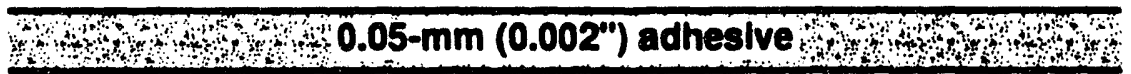

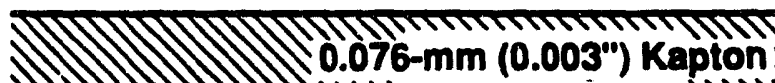

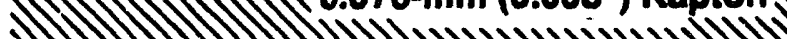

NOTE: Copper strips each 3.17-mm (0.125-in.) wide

Figure 17. Cross-section exploded end view of the twin-lead W87 cable with details of the two copper leads and associated layers of Kapton insulation and adhesive. 


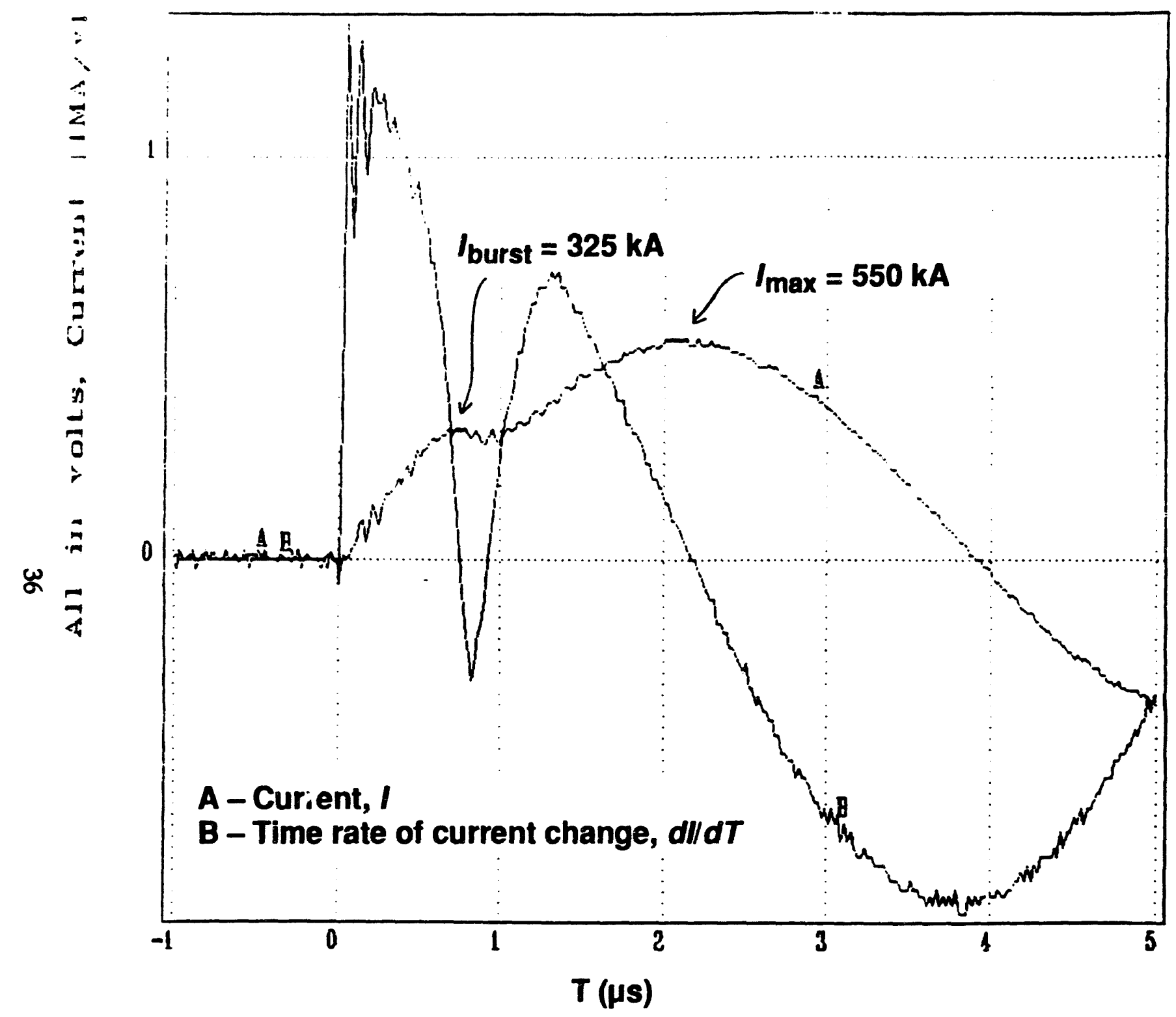

Figure 18. Waveform plots for Shot 87K119 for the case of the "both" lead connection for a 10.16-cm (4-in.) length of W87 cable. 
compared with the peak current of about $750 \mathrm{kA}$ for the much wider W89 cable. The other significant difference is the burst time of about $0.7 \mu \mathrm{s}$, which is well before current peak, while the W89 burst occurs at about $2.4 \mu \mathrm{s}$, well after current peak. The difference in current and burst times is reasonably consistent with the predictions (e.g., from the action integral or CONFUSE code) because of the differences in copper conductor widths of the two cables. For the selected bank voltage, the current at burst is about $325 \mathrm{kA}$ (nearly the same as for the W89 cable, except that in the W89 case the burst is on the backside of the current pulse, after the current peak). Table 7 lists the shots fired for measurement of the velocity and impact pressure characteristics discussed below. The Fabry-Perot velocity data for the shots were very consistent. An example of the velocitytime plot is shown in Fig. 19, including its integral, i.e., the distance the flyer traveled. At a distance of $2 \mathrm{~mm}$, the flyer is up to nearly $6.5 \mathrm{~km} / \mathrm{s}$, and at $5 \mathrm{~mm}$ it has reached $7.5 \mathrm{~km} / \mathrm{s}$ (or $\mathrm{mm} / \mu \mathrm{s}$ ). Beyond $5 \mathrm{~mm}$ or so, the Fabry-Perot laser return-light record disappeared, presumably as a result of flyer breakup.

\section{Flyer/Slapper Impact Characteristics}

As in the test series with the W89 cable, we used a witness-plate target of aluminum to investigate the footprint produced by the impact of the Kapton overcoat flyer. The standoff locations we chose for the aluminum-block witness plate were at $2 \mathrm{~mm}$ or $5 \mathrm{~mm}$ for normal impact. We studied the footprint width variation using an inclined target touching the cable at one end and the other end (of the target) at more than 5-mm standoff. The initial overcoat width of about $3.17 \mathrm{~mm}$ quickly narrowed (presumably because of the pinch effect on the driving copper plasma) to only a couple of millimeters and then slowly expanded until flyer breakup (fragmentation) beyond about $5 \mathrm{~mm}$. The narrowing in this case was not as dramatic as in the case of the W89 cable. We used the electronic streak camera was also used to observe the light produced by flyer impact on a transparent glass target. The characteristics of the flyer showed development of a concave bowed structure (highest velocity at the middle of the strip). However, neither the streak image nor the witness plate showed any distinct sidegroove structure other than the distinct edge of the strip.

\section{Impact Pressure Measurement and $\boldsymbol{P}^{2} \boldsymbol{t}$}

As in the case of the W89 test series, we employed a diagnostic target of LiF faced with $0.025-\mathrm{mm}-(0.001-\mathrm{in}$.) thick aluminum and measured the particle velocity of the $\mathrm{Al} / \mathrm{LiF}$ interface with Fabry-Perot interferometry. Then we calculated the time-resolved impact pressure just inside the LiF target, using the known EOS for the LiF and the particle velocity history. In the first experiments, however, we lost the Fabry-Perot trace even before impact. We then realized that the target at 2- to $5-\mathrm{mm}$ standoff was close enough for the eddy current heating of the increasing current in the cable possibly to melt and destroy the reflectivity of the thin aluminum (face) coating. We then installed a simple electromagnetic shield just above the test cable section with an aperture through which the flyer/slapper could easily pass. The Fabry-Perot fringes could then be 
Table 7. Shot $\log$ of $\mathbf{W} 87$ detonator cable tests in $87-\mathrm{kJ}$ electric gun at $60 \mathrm{kV}$.

\begin{tabular}{|c|c|c|c|c|c|c|c|}
\hline Shot\# & Geometry & $I_{(\max )}(K A)$ & $\mathbf{I}_{\text {(burst) }}(\mathrm{KA})$ & $V_{f}(2 \mathrm{~mm}) \mathrm{km} / \mathrm{s}$ & $V_{f}(5 \mathrm{~mm})(\mathrm{km} / \mathrm{s})$ & $P_{(\max )(G P a)}$ & $\int \mathrm{P}^{2} \mathrm{dt}\left(\mathrm{GPa}^{2} \mu \mathrm{s}\right)$ \\
\hline 113 & 4-in. $(10.16 \mathrm{~cm})$ "both" & 480 & 305 & 6.4 & 7.5 & & \\
\hline 114 & 4-in. "both" & 492 & 317 & $>6.0$ & Timing shot & & \\
\hline 115 & 4-in. "both" & 526 & 323 & Poor impact, pres & sure pulse disappear & red from F-P rec & cord \\
\hline 116 & 4-in. "both" & 525 & 331 & $>6.0^{\circ}$ & Timing shot & & \\
\hline 117 & 4-in. "both" & 528 & 328 & Poor impact, pres & sure pulse disappear & red & \\
\hline 118 & 4-in. "both" & 538 & 331 & Poor impact, no $\mathrm{F}$ & ressure pulse recorde & & \\
\hline 119 & 4-in. "both" & 549 & 325 & The pressure puls & se too poor to be meas & surable & \\
\hline 120 & 4-in. "both" & 531 & 328 & $\begin{array}{l}\text { Again no pressur } \\
\text { NOTE: Magnetic }\end{array}$ & $\begin{array}{l}\text { e pulse recorded } \\
\text { shielding installed on }\end{array}$ & n Shot 120 & \\
\hline 121 & 4-in. "both" & 550 & 333 & & & $43 \mathrm{GPa}$ & $10 \mathrm{GPa}^{2} \mu \mathrm{s}$ \\
\hline 123 & 4-in. "both" & 623 & 339 & & & $45 \mathrm{GPa}$ & \\
\hline
\end{tabular}




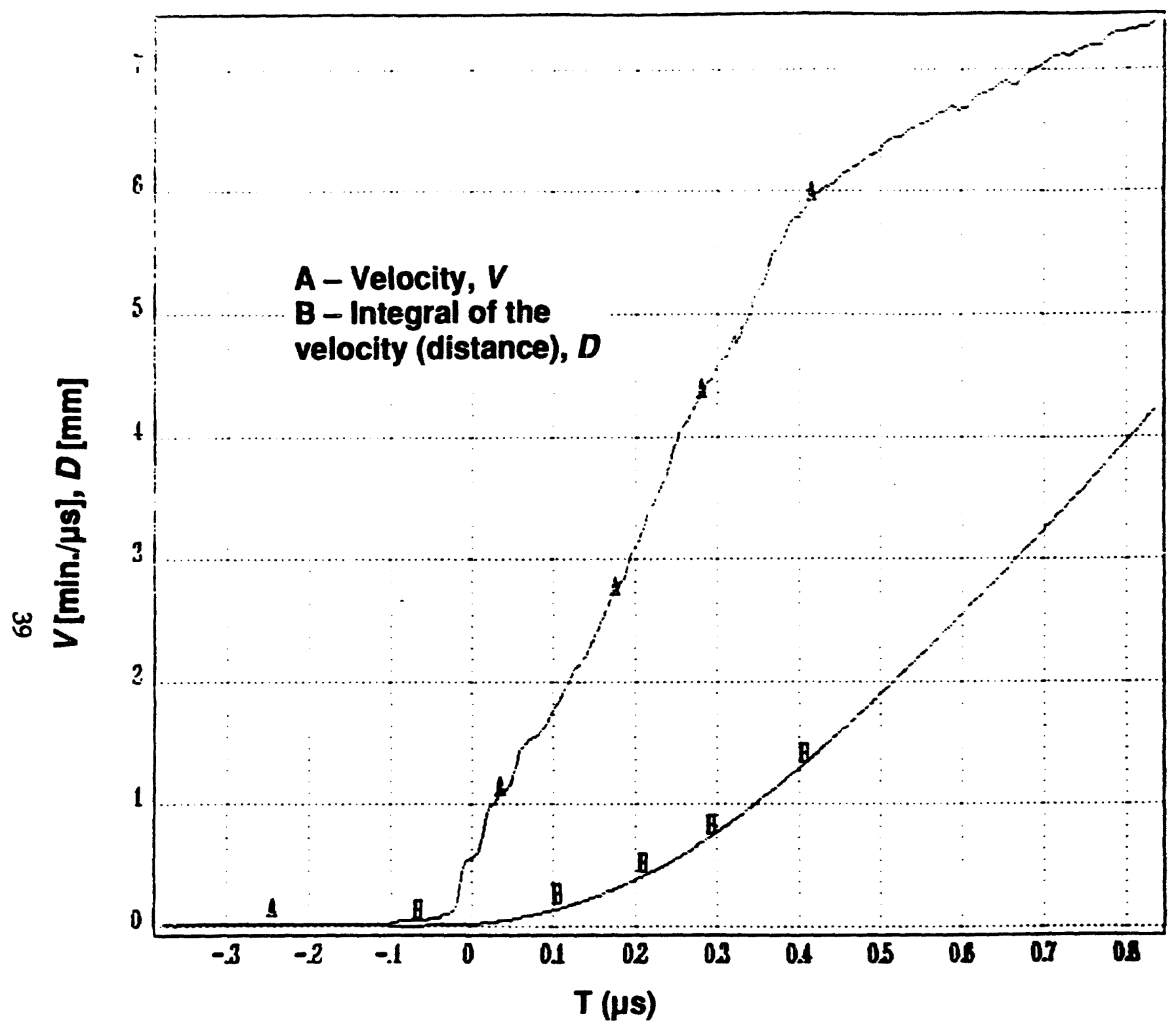

Figure 19. Plot of Shot $87 \mathrm{~K} 113$ cover-layer "flyer" velocity history and integral for a $10.16-\mathrm{cm}(4-\mathrm{in}$.) section of $\mathrm{W87}$ cable energized at a level of $31 \mathrm{KJ}(60 \mathrm{kV})$ for the case of "both" initial connection. The integral of the velocity shows the distance traveled as a function of time. 
followed and the pressure (and the integral of pressure over time) measured, as shown in Fig. 20. The corresponding value of $P^{2}$, with its time integral used as a measure of $P^{2} t$, is shown in Fig. 21.

\section{Discussion}

The measured flyer velocities are typically above thresholds determined in Ref. 5 for the detonation of either LX-17 or UFTATB. However, the strip width is now much narrower than the minimum critical spot size expected for detonation of even hot LX-17. Again, as in the discussion of the W89 cable test series, the pressure values obtained using the LiF technique were lower than those expected from impact of fulldensity Kapton at the measured velocity. The two most likely explanations include heating of the Kapton by copper plasma such as to modify the EOS of the Kapton or reading errors in the very sharply spiked pressure pulses from impact of the very thin Kapton flyer $0.076-\mathrm{mm}$ - $(0.003$-in.) thick Kapton and perhaps $0.05-\mathrm{mm}$ - $(0.002$-in.) thick softer adhesive). The Fabry-Perot as used has a time resolution of a few nanoseconds at best, so the pressure measurement is subject to a relatively large error bar.

The result from these velocity and $P^{2} t$ test measurements is that the margin of safety of the W87 system in an abnormal electrical environment also depends on the minimum critical spot size for detonating hot LX-17 or UFTATB. The impact strip width is much narrower than the spot size for ambient $L X-17$, but tests should be performed to extend the critical spot-size criteria for strip flyers impacting $250^{\circ} \mathrm{C} L X-17$ and UFTATB. 


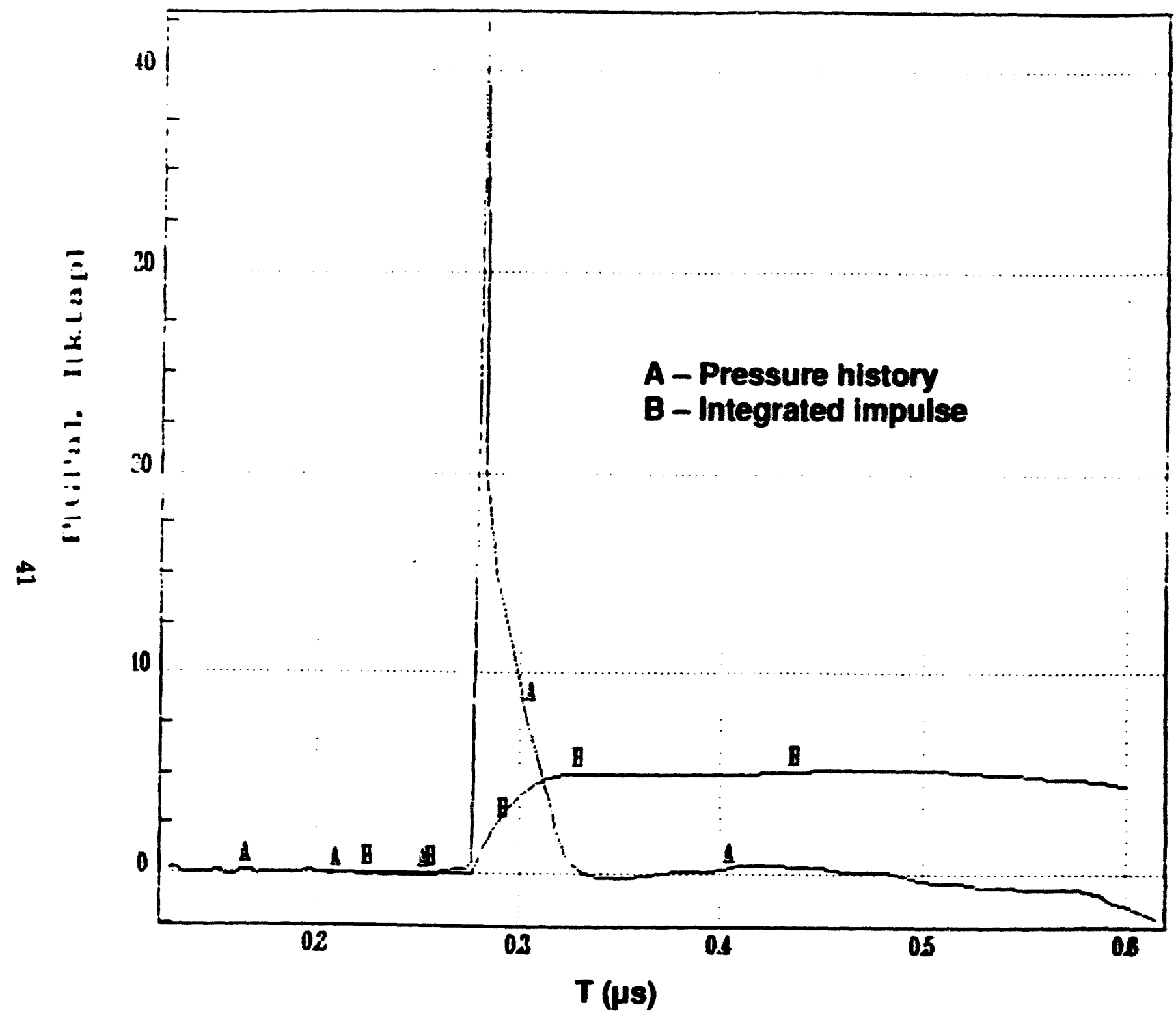

Figure 20. For Shot 87K121, the pressure history and its integrated impulse for a "both" lead test case. 


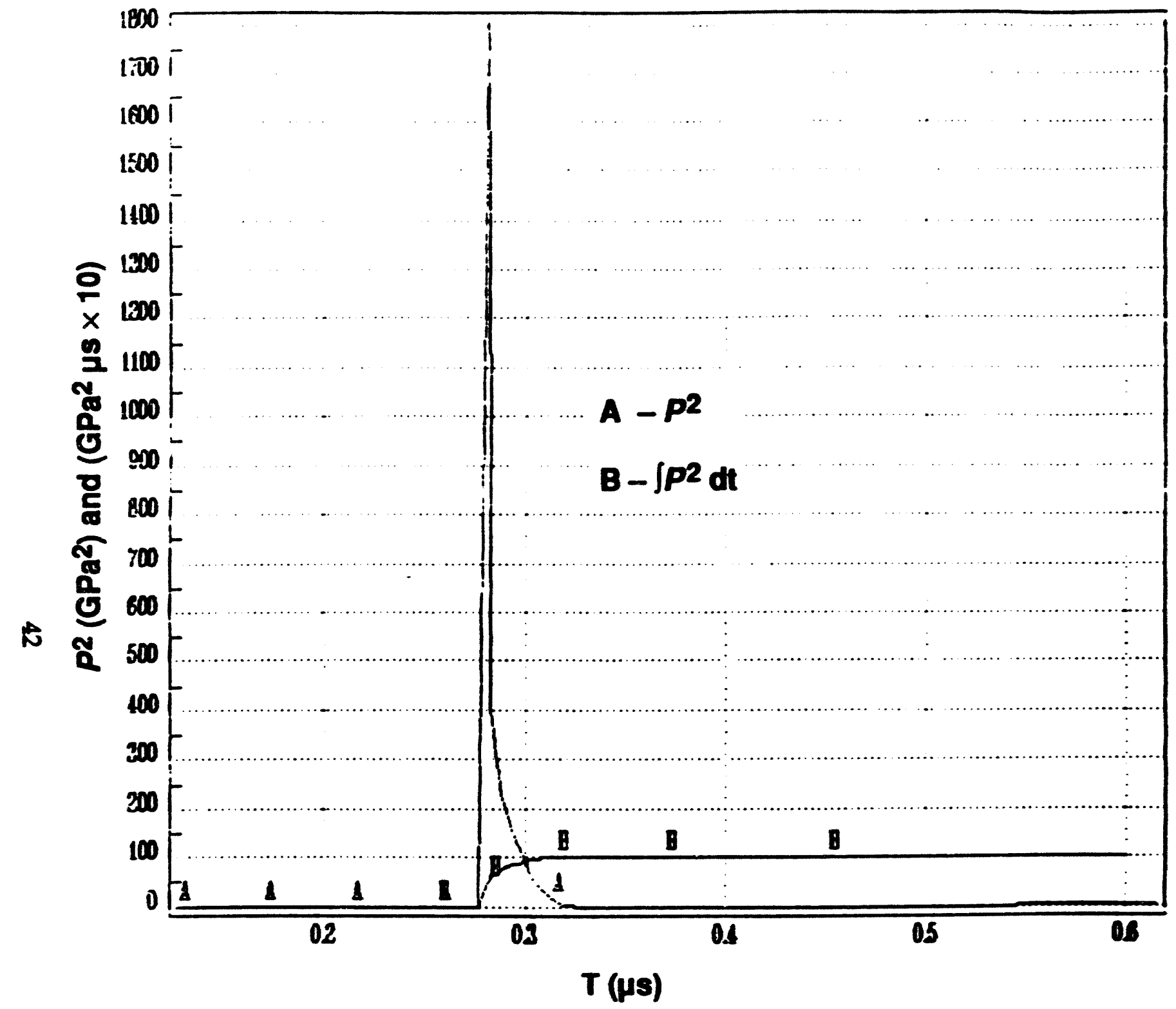

Figure 21. For Shot 87K121, the pressure-squared history (pressure shown in Fig. 19) and its time-integrated value are shown as an estimate of $\mathrm{P}^{\mathrm{q}} \mathrm{\text {(value }}$ taken at about 0.1 $\mu$ after impact, to avoid spurious late-time signals from edge reflections in the LiF crystal). 


\section{GLOSSARY}

DSSL

EOS

Fabry-Perot

FPC 461

HE

HEAF

HMXX

IHE

KeL-F 800

Kapton

Lexan

LiF

LX-10

LX-17

MSAD

PBX-9407

PBX-9501

PBX-9502

RDX

TATB

Ultrafine TATB

Viton A
Detonator safing strong link

Equation of state

Inth rferometry technique

Binder; Vinyl Chloride/Trifluorochloroethylene/Copolymer

\section{$1.5: 1$}

High explosive

High Explosives Applications Facility

1, 3, 5, 7 - Tetranitro-1, 3, 5, 7 - Tetrazacyclooctone

Insensitive high explosive

Binder; Chlorotrifluoroethylene/Vinylidine Fluoride

Polyimide film $\left(\mathrm{C}_{22} \mathrm{H}_{10} \mathrm{~N}_{2} \mathrm{O}_{5}\right)$

Trade name for polycarbonate plastic

Lithium fluoride

LLNL material; HMX/Viton composition $95 / 5 \%$

LLNL material; TATB/KeL-F 800 composition $92.5 \% / 7.5 \%$

Mechanical safing and arming device

LANL material; RDX/FPC 461 composition 94/6\%

Ref. on p. 3

LANL material; TATB/KeI-F 800 composition 95/5\%

Hexahydro-1, 3, 5 - Trinitro - 1, 3, 5-Triazine

1,3,5 - Triamino-2, 4, 6 - Trinitrobenzene

Pure TATB; $\rho=1.8 \mathrm{~g} / \mathrm{cm}^{3}, 10-\mu \mathrm{m}$ mean particle size

Binder; Vinylidine Fluoride/Hexafluoropropylene 
$1 !$
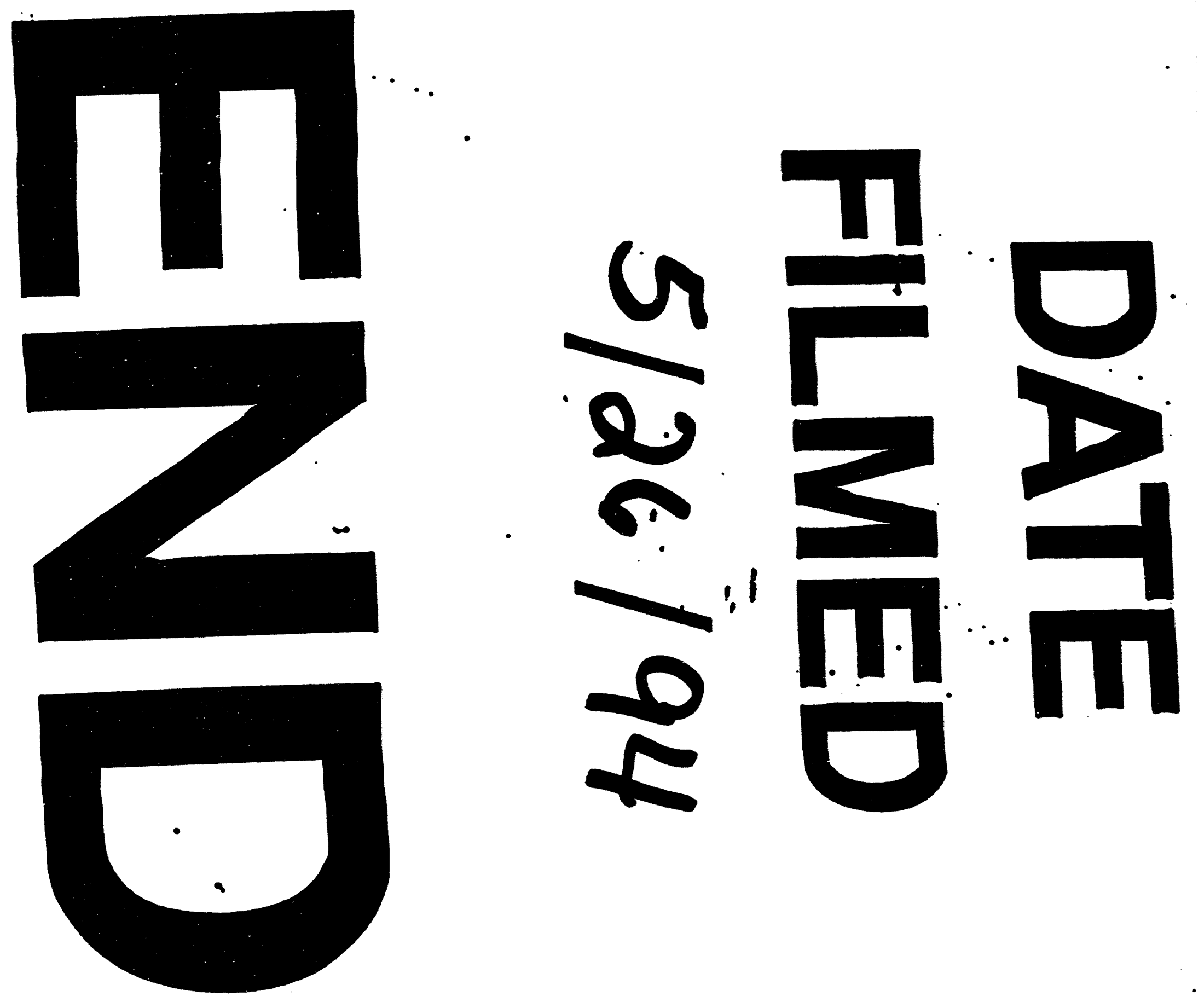
